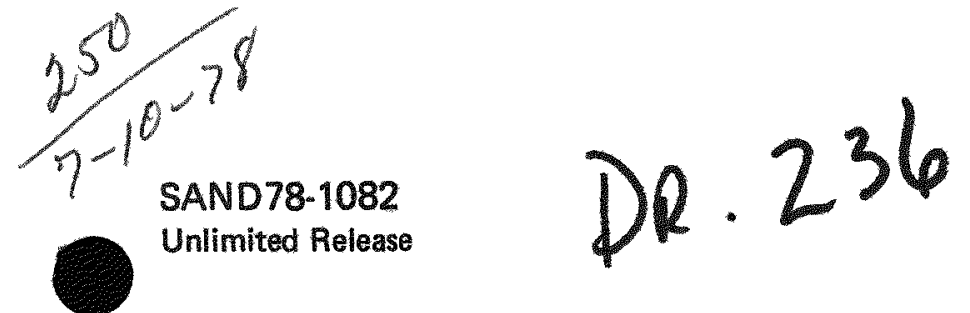

\title{
Product Identification File
}

Charles E. Gray, Editor

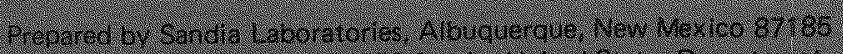

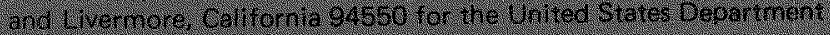

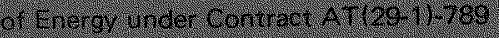

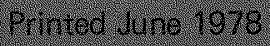

Sandia Laboratories 
Issued by Sandia Laboratories, operated for the United States Department of Energy by Sandia Corporation

NOTICE

This report was prepared as an account of work sponsored by the United States Government. Neither the United States nor the United States Department of Energy, nor any of their employees, nor any of their contractors, subcontractors, or their employees, makes any warranty, express or implied, or assumes any legal liability or responsibility for the accuracy, completeness, or usefulness of any information, apparatus, product or process disclosed, or represents that its use would not infringe on privately owned rights.

Printed in the United States of America

Available from

National Technical Information Service

U. S. Department of Commerce

5285 Port Royal Road.

Springfield, VA 22161

Price: Printed Copy $\$ 6.00$; Microfiche $\$ 3.00$ 


\section{DISCLAIMER}

This report was prepared as an account of work sponsored by an agency of the United States Government. Neither the United States Government nor any agency Thereof, nor any of their employees, makes any warranty, express or implied, or assumes any legal liability or responsibility for the accuracy, completeness, or usefulness of any information, apparatus, product, or process disclosed, or represents that its use would not infringe privately owned rights. Reference herein to any specific commercial product, process, or service by trade name, trademark, manufacturer, or otherwise does not necessarily constitute or imply its endorsement, recommendation, or favoring by the United States Government or any agency thereof. The views and opinions of authors expressed herein do not necessarily state or reflect those of the United States Government or any agency thereof. 


\section{DISCLAIMER}

Portions of this document may be illegible in electronic image products. Images are produced from the best available original document. 
SAND78-1082

Unlimited Release

Printed June 1978
Distribution

Category UC-4I

PRODUCT IDENTIFICATION FILE

Compiled and Edited by Charles E. Gray Environmental Health Laboratory, 3311

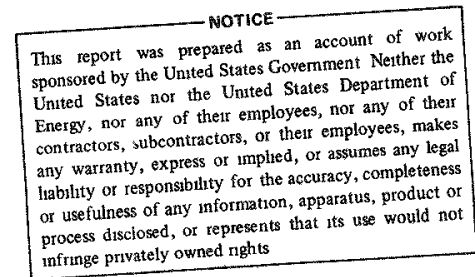

Environmental Health Department, 3310

Sandia Laboratories

Albuquerque, New Mexico 87185 

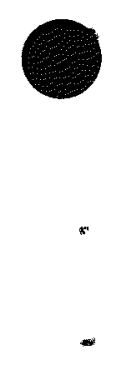

○

1 


\section{PREFACE}

The Product Identification File has been compiled and edited by Charles E. Gray, 3311 Chemistry Laboratory, as an aid to the industrial hygienist who may encounter the products listed in surveys for and studies of occupational health hazards.

It should be remembered that the chemical composition of a product may vary from year to year and some components may be added or deleted without an indication on the label. Some of the data in this file may not be complete depending upon the analysis requested. For example, a solvent may be the only component for which the product was analyzed.

This file is arranged by listing the chemical manufacturer followed by the trade name. In cases where no manufacturer is known, the trade name appears in alphabetical order.

The log number and the chemist who analyzed the product are listed for reference by the Chemistry Laboratory. 
OTHER SOURCES FOR DATA ON TRADE NAME PRODUCTS

Reference sources are listed below. Those references preceded by an asterisk list some chemical component data. The other references give trade names and addresses of manufacturers.

American Man-Made Fiber Trade Names. Mod. Textiles Mag. 45:54 (Mag. 1964).

*Chemical Toxicology of Commercial Products, 4th Edition, Marion N. Gleason, et al., Williams and Wilkins Co., Baltimore, Maryland, 1976.

*Chemical Trade Names and Commercial Synonyms, William Haynes, D. Van Nostrand Co., New York, New York, 1951.

*Chemical Synonyms and Trade Names, William Gardner, et al., CRC Press, Cleveland, Ohio, $1 \overline{968 .}$

Condensed Review of Some Recently Developed Materials; Arranged by Trade Names. Mach. 70:123 (Oct. 1963).

Conover-Mast Purchasing Directory, 205 E. 42nd St., New York 17, New York.

*Directory of Chemicals and Producers, Wallace F. Traendly, and Thomas I. Bonnitt (E)itors). McGraw-Hill Publishing Co., 330 w. 42nd St., New York 18, New York, 1952.

Electrical Products Guide; Company and Trade Names. Elec. Constr. \& Maint. 60:43 (Mid-Sept. 1961).

1964 Equipment Directory; Trade Names. Mod. Materials Handling, 19:71 (May 1964).

Guide to Tool Steels and Carbides. Steel 148:99 (May 15, 1961).

*Handbook of Material Trade Names, 0. T. Zimmerman and Irvin Lavine (1946, 1953). Sup. I 1956; Sup. II 1957; Sup. III 1960. Industrial Research Services, Inc., Dover, New Hampshire.

*Handbook of Plastics. Herbert R. Simonds and Carleton Ellis. D. Van Nostrand Co., Inc., New York, 1943.

*Handbook of Solvents. Jacob Scheflan. D. Van Nostrand, Co., Inc., New York, 1953.

MacRae's Blue Book (annual). Trade Names Section, MacRae's Blue Book Co., $18 \mathrm{E}$. Fluron St., Chicago 11, Illinois. 
Materials Handling and Packaging Directory; Trade Names. Mod. Materials Handling 18:69 (May 1963).

*The Merck Index, 9th Edition, Merck \& Co., Rahway, New Jersey, 1976.

Modern Plastics Encyclopedia Issue (annual). Plastics Catalogue Corp., Emmet st., Briston, Connecticut.

*Paint Trademark Directory (triennially). Trademark Bureau, Nat. Paint, Varnish and Lacquer Association, Inc., 1500 Rhode Island Avenue, N.W., Washington 5, D.C.

*Pesticide Index, D. E. H. Frear, College Science Publishers, P. O. Box 798, State College, Pa., 1961.

Plastics World Contemporary Film and Sheeting. Property and Price Chart; List of Trade Names. Plastics World 19:46 (Apr. 1961).

* Sadtler Standard IR Commercial Spectra, Sadtler Research Laboratories, Inc., Philadelphia, PA, 1974 .

Textile World Fact File; Trade Name Index. Textile World 111:315 (MidJuly 1961).

Trade Names of Cosmetic Materials by Product Classification. Am. Perfumer and Aromatics, Documentary Ed., p. 271 (1960).

Trade Names; Processes, Equipment and Materials Used in the Water Supply and Treatment and their manufacture. Water Works Eng. 116:406 (May 1963).

*Trade Names Index, Chem. Eng. 69:223 (Nov. 1962).

Trade Name Index; Welding Tools and Supplies. Welding Eng. 48:124 (Midyear 1963).

Trade Name Index; Products in or Related to the Field of Electronics. Electronics 36:175 (July 25, 1963).

* Trade Names Index; published by the American Conference of GovernmentaI Industrial Hygienists, 1964.

*Trade Names Index; published by the American Conference of Governmental Industrial Hygienists, Supplement No. 1, 1966.

* Trade Names Index; published by the American Conference of Governmental Industrial Hygienists, Supplement No. 2, 1968.

Two other sources for information on chemical composition of trade name products are: (1) Scientific Reference Services Branch, Division of 
Occupational Injury and Disease Control, Bureau of Occupational Safety and Health, Environmental Control Administration, Consumer Protection and Environmental Health Service, 1014 Broadway, Cincinnati, Ohio 45202

(513:684-2963), and (2) Pois on Control Centers of State, local health departments or major universities. 


\section{ACKNOWLEDGEMENTS}

Acknowledgement is made of the assistance of various chemists in the Environmenta. Health Chemistry Laboratory during the last ten years; namely, Messrs. Lial W. Brewer, Wayne Einfeld, Grover Edwards, John Grillo and Ms. Ednarae Gross. Recognition is hereby given to the work of Susan Kissam for her untiring efforts in performing much of the clerical work and typing for this Product Identification File. 

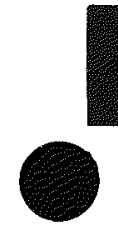

- 
Accelerator 808

Contains butyraldehydebutylamine

Acrylate Solvent

Contains ethylene glycol monoethyl ether and

Log \#761107

a mixture of xylenes.

Addressograph Multigraph Corp., East Hanover, NJ

Varityper 3020 Developer

Contains borax or boric acid, sodium sulfite or bisulfite, hydroquinone; $\mathrm{pH}$ of $1 \%$ solution $=8.8$.

Addressograph Multigraph Corp., East Hanover, NJ

Varityper 3025 Fixer

Mixture of boric acid, sodium aluminum sulfate

and sodium thiosulfate; $\mathrm{pH}=7.3$.

Addressograph Multigraph Corp., East Hanover, NJ

Varityper 3225 Tank Cleaner

$\mathrm{pH}<1$; sodium chromate or dichromate in dilute

sulfuric acid.

Adheron Epoxy Emulsion Coating

Emulsion is white pigmented low mol. wt. epoxy

CEG

Log \#750317

CEG

resin. (No unsaturation remaining.) Only volatile

Log \#750318

$\mathrm{CEG}$ solvents are water and $\mathrm{C}_{5}-\mathrm{C}_{10}$ st. chain hydrocarbons. Main solvent $(>90 \%)$ is water. Curing agent is a planer secondary polyamide with no volatile organic solvents. In mixed form a copolymer (epoxy-polyamide) is formed.

Ads orbite

Activated charcoal.

Aero-Carb E

Heat treating salts. Contains sodium cyanide.

Aero-Heat 300

A mixture of sodium and potassium nitrates and nitrites. For heat treating metal.

Age Rite Gel

Octylated diphenylamine

Age Rite Hypar

Phenyl beta naphthylamine

Age Rite Powder

Pheny 1 B-naphthyI amine 
Age Rite Resin

Aldol naphthylamine

Age Rite Resin D

Trimethyl dihydroquinoline

Age Rite Stalite

Octylated diphenylamine mixture

Age Rite Superflex

Diphenylamine acetone

Aircosil

Silver Brazing Alloy

\#45 23.3\% Cd (TNI 23 - 25\%)

Log \#660532

JPG

Akroflex C

Phenyl alpha napthylamine

Albi Manufacturing Corp., Rockville, CT

Albi-144 Fire \& Heat Retardant Coating

Contains alkyd resin. Solvents are aliphatic

Log \#770732

CEG

hydrocarbons. Also contains unreacted phthalic

anhydride in the solvent. Contains urea phosphate and possibly some ammonium phosphate as the fire retardant. (Bubbles are formed when heated.)

Albi Manufacturing Corp., Rockville, CT

Fire Protection Coating \#160B Trowel Grade

Solvent is water. Glass fibers present.

$\mathrm{P}, \mathrm{Si}, \mathrm{B}, \mathrm{Mg}$, Ti, $\mathrm{Na}, \mathrm{Al}, \mathrm{Ca}, \mathrm{Fe}$ present.

Log \#772595

$\mathrm{CEG}$

Albi Manufacturing Corp., Rockville, CT

Albi-R Fire Resistant Paint

Contains formaldehyde

Albuquerque Duplicator Supply, Albuquerque, NM

Imperial Duplicator Fluid

Log \#670251

$\mathrm{CH}_{3} \mathrm{OH}$. Direct process spirit type high methyl

LWB

alcohol content equivalent to Type II Fed. Spec.

0.D-825. $N_{D}=1.3325$ (18); flash point $49^{\circ} \mathrm{F}$ OC;

TLV $200 \mathrm{ppm} ; \mathrm{mg} / \mathrm{m} 3 ; 20^{\circ}-70^{\circ} \mathrm{F}$ flammability.

Alcohol, Denatured, Toxic

Toxic - see methyl alcohol, as issued from

General Stores (2-1-62); 4 gal. methyl

isobutyl ketone; 1 gal kerosene; $1100 \mathrm{gal}$.

190 proof ethyl alcohol.

Aliquat 215

Dioleyl-linoleyl dimethyl ammonium chloride. 
All Safe Waterless Handcleaner

Deodorized kerosene plus lanolin and silicone

$\log \# 661577$

LWB

Allstate

Solder \#105

98\% cd. (TNI 95\%)

Log \#660574

EG

Allstate

430 Flux

$<0.05 \% \mathrm{Cd}$. Strongly acidic aluminum and a.lkali metal chlorides. $\mathrm{ZnCl}_{2}-\mathrm{NH}_{4} \mathrm{Cl}, \mathrm{CH}_{3} \mathrm{OH}$, ethylene gycol.

Log \#661527 \& 650341

JPG

Almag Cutting Oil

A petroleum based light oil. Flash point $295^{\circ} \mathrm{F}$ $\mathrm{OC} ; 628 \mathrm{~mm} / \mathrm{Hg}$.

Log \#650233

LWB

Almandite

Ferric aluminum silicate used by Org. 1113.

$12 / 15 / 64$

Alpenco Lens Cleaner \& Anti-Fogging Liquid

Flash point $74^{\circ} \mathrm{F}$ at $625.95 \mathrm{~mm} / \mathrm{Hg}$.

$7 / 8 / 64$

A Itax

Benzothiazyl disulfide

Aluminate Abrasive \#I

$50.3 \%$ free silica $+\mathrm{Al}_{2} \mathrm{O}_{3}$ of $27 \mu$ size. (The

Log \#660206 purity is questionable.)

Aluminon

Ammonium salt of aurix tricarboxylic acid.

Aluminum detection and estimations.

Alumon

A zincate powder, proprietary product. Used to make a solution for coating aluminum with a zinc finish prior to electroplating other metals on the aluminum.

American Cyanamid

Aero-Seal \#52

43\% polyethylene 0-phthalate resin. Balance is

Log \#680546 $\mathrm{H}_{2} \mathrm{O}$. (Water emulsion)

JPG

American Lumber \& Treating Co.

Wolmanized Lumber

Contains dinitrophenol. Preservative poisonous when taken internally; may cause dermatitis to sensitive people. 
Aminox

Diphenylamine acetone.

Ampex Head Assembly Cleaner

Xylene

Analabs

$3 / 18 / 64$

Anakrom

Gas chromatograph column flller (J. Hesse, Org. 5135).

Apeco Auto-Stat 1-Step Concentrate

Boiled at $200^{\circ} \mathrm{F}, 626.65 \mathrm{~mm} / \mathrm{Hg}$ without flashing.

$5 / 11 / 64 \& 5 / 26 / 64$

Boiled at $185^{\circ} \mathrm{F}, 625.65 \mathrm{~mm} / \mathrm{Hg}$ without flashing, $5 / 26 / 64$.

Arcal

Arcal Weld-O Aluminum Brightener

$6.0 \% \mathrm{HF}, 10 \%$ alkyl aryl polyglycol ether, balance $\mathrm{H}_{2} \mathrm{O}$.

Armstrong

Armstrong Epoxy Stripper \#44

Stripper is trichloroacetic acid in a saturated chlorinated hydrocarbon solvent (possibly

dichloroethane). Small amount of water. pH of $1 \%$ soln. $=2.4$.

Log \#673374

$\mathrm{JPG} / \mathrm{EG}$

Log \#701285

CEG

Armstrong

Armstrong Stripper

Methylene chloride plus some phenol. 4/7/64, ref.

N. J. DeLollis, 1112-2.

Armstrong Cork, Lancaster, PA

Armstrong Cement S-1200

Cement solvent contains acetone and petroleum ether plus some minor other aliphatic organics.

No aromatic or chlorinated solvent present.

Armstrong Cork, Lancaster, PA

Armstrong $E$ Activator

DEAPA-3-diethylaminopropylamine

Armstrong Products Co., Warsaw, IN

Armstrong Stripper \#49

$83.4 \%$ methylene chloride, $14.1 \%$ acetic acid,

$2.5 \%$ sodium acetate.

Athea Laboratories, Milwaukee, WI

Athea Hand Lotion

Log \#762394

Contains petroleum, lanolin, veg. oil, stearic

CEG

Log \#630323

TAL

Log \#680406

JPG

acid, hexadecanol and propylene glycol. $4 \%$

$\mathrm{H}_{2} \mathrm{O}, \mathrm{pH}=7.0$. 
AYA Blends

Bakelite polyvinyl acetate polymers, emulsified in

$\mathrm{H}_{2} \mathrm{O}$. One of few water soluble adhesives. Harmless, is used in food wrappings and adhesives such as Elmer's glue.

Azograph Duplicating Fluid

Pure methanol; flash point $47^{\circ} \mathrm{F}$.

Azrock Cut-Back Adhesive

$12 / 21 / 62$

Flash point $49^{\circ} \mathrm{F}$ CC, MC. 
Baker Chemical Co.

Log \#670208

K.P.R. Stripper

$85 \% \mathrm{CH}_{2} \mathrm{Cl}_{2}$ (methylene chloride); $10 \%$ toluene

JPG

$\left(\mathrm{C}_{6} \mathrm{H}_{5} \mathrm{CH}_{3}\right) ; 5 \%$ unknown - probably a wetting agent.

Ball Powder Double Base

See nitrocellulose and nitroglycerin.

Baratol

See trinitrotoluene and barium nitrate.

Bayflex Wheels

Cotton fibers, abrasives, phenolic resin, neoprene, latex and casein. The abrasive used is aluminum oxide $\left(\mathrm{Al}_{2} \mathrm{O}_{3}\right)$ (man-made).

Beck Chemical Co., Cleveland, $\mathrm{OH}$

Epoxy Strip Type T-2Sl-C

Contains a mixture of cresols or phenols, chloroacetic acid and formic acid.

Bell \& Howell

HC-300 Etchant

Contains $\mathrm{Fe}$ and $\mathrm{Cr}$ and $\mathrm{H}_{2} \mathrm{SO}_{4} ; \mathrm{pH}$ of conc.
solution $<0.1$.

Log \#730706

$\mathrm{CEG}$

Log \#710734

CEG

Log \#741004

Bestline Co.

CEG

Major component is water. Minor components are

iso-propyl alcohol, ammonia and an anionic

surfactant. $\mathrm{pH}=10$.

Best Products, Inc.

"Zif" Cleaner

Log \#722742

$\approx 9-12 \%$ alkyl aryl sulphonic acid; $\approx 4 \%$ diethanolamine;

$\approx 12 \%$ phosphates; $\approx 4 \%$ silicates; and $70 \% \mathrm{H}_{2} \mathrm{O} . \mathrm{pH}=9.5$.

$\mathrm{BFC}$

Vinyl Thinner (Beta Cote)

100\% N-butyl acetate (commercial grade).

$\log \# 661502$

LWB

Bison - 1700 Lubricant

$\mathrm{pH}=6.9$

Log \#630062

TAL 
Blair Art Products, Memphis, TN

Log \#762060

Blair Spray Fix

Contains $2 \%$ methyl alcohol; $3 \%$ ethyl alcohol;

CEG

15\% acetone; $13 \%$ methyl acetate; $12 \%$ methylene chloride; 30\% MEK; 7\% butyl alcohol; $18 \%$ butyl acetate.

Blanket and Roller Wash

$50 \%$ tetrachloroethylene and 50\% petroleum

$4 / 18 / 74$

distillate (aliphatic HC). Flash point

$110^{\circ} \mathrm{F} \mathrm{OC,} 78^{\circ} \mathrm{F} \mathrm{CC}$.

Blanket Roller Wash \#595458

Contains $50 \%$ perchloroethylene, $50 \%$ aliphatic

$\log \# 740670$

hydrocarbon.

CEG

Blankrola

$3 \%$ rosin oil and 97\% coal tar naptha. (This product now contains some perchloroethylene 4/17/70 - D. Parker.)

Borax Co.

Borax Hand Soap \#221

Contains B, Si, $\mathrm{Na}$; $\mathrm{pH}=8$.

Log \#760880

CEG

BPI Print Flattening Solution

Boiling point $175^{\circ} \mathrm{F}, 627 \mathrm{~mm} / \mathrm{Hg}$ without flashing.

Andrew Brown Co.

Brolite Synflex H-22 Clear Baking Enamel

$27.2 \%$ clear enamel; $49.0 \%$ toluene; $12.2 \%$ xylene ( $10.2 \%$ o, $\mathrm{m} ; 2.0 \% \mathrm{p}) ; 8.0 \%$ isobutyl alcohol; $4.0 \%$ isobuty 1 ether; $2.3 \%$ water; $1.0 \%$ cellosolve acetate.

Brunswich Chemical Co., Chelmsford, MA

Log \#680588

Den-centrate Copier

$40 \%$ carbon black; $60 \%$ high-boiling aliphatic hydrocarbons, including paraffin. Flash point $>235^{\circ} \mathrm{F} \mathrm{OC}$.

Brunswick Chemical Co., Chelmsford, MA

Log \#680980

JPG

Den-toner High Speed Copier

$100 \%$ high-boiling hydrocarbons; flash point $127^{\circ} \mathrm{F}$ $\mathrm{OC}$.

Buehler $A B$ Lapping Oil

Flash point $190^{\circ} \mathrm{F}$ at $621.05 \mathrm{~mm} / \mathrm{Hg}$. 
Cadet Chemical Co., Burt, NY

Cadox MDP

Methyl ethyl ketone peroxide.

Cadox TS -5

$50 \%$ 2,5-dichloro-benizoyl peroxide in silicone.

Calgon Skin Care Lotion

$1.5 \%$ triethanol amine, $0.02 \%$ lanolin.

Log \#770828

CEG

Captax

Mercaptobenzothiazole.

Carbitol

Diethylene glycol mono ethyl ether.

Carboline Co.

Fire Protection Coating \#I-147-172-50

Log \#772988

Solvent is water. Glass fibers present. P, Si,

CEG

$\mathrm{B}, \mathrm{Mg}, \mathrm{Ti}, \mathrm{Na}, \mathrm{Al}, \mathrm{Ca}, \mathrm{Fe}$ present.

Carrene Refrigerant

Same as Freon 11, trichloromonofluoromethane.

Carthane

Urethane foam resin.

Century Papers, Inc., Houston, IX

Super Mr. Jon Instant Bowl Cleaner

$\mathrm{pH}$ of undiluted product is 1. Percent of

Log \#770133

CEG

$\mathrm{HCl}=27.5$.

Certified Laboratories, Ft. Worth, TX

"WAS-16" Anti-Splatter Compound

Lightweight aliphatic hydrocarbon oil with a

Log \#730007 mixture of $\mathrm{CO}_{2}$ and nitrous oxide as propeliants.

$\mathrm{CEG}$

Chemtron Corp., Downey, CA

CEE BEE

Contains > 50\% methylene choride; < $20 \%$ phenol;

Log \#752028

CEG $<20 \%$ formic acid.

Chevron

RPM Delo Motor Oil

Log \#772718

Material is an aliphatic hydrocarbon oil. 
Chevron

RPM Hydraulic Oil

Flash point $215^{\circ} \mathrm{F} \mathrm{OC,} \mathrm{41-63} \mathrm{MC.}$

Chlorothene (Vythene) 500 ppm

Methylchlor oform compound, degreasing solvent.

See 1,1,1-trichloroethane.

ChIorothene Solvent

94-97\% 1,1,1-trichloroethane, 2.4 to $3 \%$ dioxane, 0.12 to $.3 \%$ butanol and small amounts of ethylene dichloride, water and other materials. Ind. Hyg. J., Aug. 6I, p. 253.

Chromerge

Chromium salt and sulfuric acid cleaning solution.

Cincinnati Milling Machine Co.

Cim Cool 30

$\log \# 762316$

Contains nitrites.

Cincinnati Milling Machine Co.

$\log \# 741001$

Cim Perial \#15 Cutting Oil

Material is an aliphatic hydrocarbon oil. No aromatics present. Contains a trace of formaldehyde. $\mathrm{pH}=9$ conc. form.

Cincinnati Milling Machine Co.

Cim Plus 49

Contains nitrites.

Log \#762317 $\mathrm{CEG}$

Clearco Products Co., Philadelphia, PA

Welding Jelly

Emission spectra shows the elements $\mathrm{C}, \mathrm{Si}, \mathrm{Fe}$.

Log \#730028

I.R. indicates a di-phenyl silicone polymer

and a high molecular weight hydrocarbon oil.

Cobehn Co., Fairfield, NJ

Cobehn Spray Cleaning Solvent

Chloroform $+\sim 1 \%$ ethyl alcohol. (96\% $\mathrm{CHCl}_{3}+$

4\% EtOH (IASI 4/20/73) Ref. Bill stocum.)

Codo Manufacturing Corp.

Duplicating Fluid

$90 \% \mathrm{CH} 30 \mathrm{H}, 10 \%$ cellosolve, contains wood alcohol.

Log \#670252

Conforms to Type II Fed. Spec. O-D-825. Flash

point $48^{\circ} \mathrm{F}$ OC, flammability $20-70^{\circ} \mathrm{F} . \quad N_{D}=1.3345$

(18).

Collodion

A solution of nitrocellulose in ethyl alcohol

and ether. If it also contains camphor, it

becomes celluloid when it is dried. Very

flammable; flash point $-49^{\circ} \mathrm{F},-45^{\circ} \mathrm{C}$.

$10 / 3 / 61$

$\mathrm{JE}$

LWB 
Colorado Paint Co.

Material is linseed oil.

Columbia Ribbon \& Carbon Mfg. Co., Inc., Aurora, IL Duplicating Fluid

Log \#670248

$\mathrm{CH}_{3} \mathrm{OH}-90 \%+10 \%$ ethyl cellosolve; for use on all

LWB splrit duplicating machines. $\mathbb{N}_{D}=1.3280$ (18). Flash point $49^{\circ} \mathrm{F}$ OC; flammability $20-70^{\circ} \mathrm{F}$. Toxicity hazards - TLV $200 \mathrm{ppm} ; \mathrm{mg} / \mathrm{m}^{3}$.

Columbium Carbon Co.

Neatax

Carbon black.

Copytron Toner

Purple thermoplastic resin

$\log \# 641525$

LWB

Corona Chromium Etchout

Contains $\mathrm{Fe}$ and $\mathrm{Cr}$ and $\mathrm{H}_{2} \mathrm{SO}_{4} \cdot \mathrm{pH}$ of conc.

Log \#710735 solution $=0.1$.

CEG

Crown Toolmakers Ink Remover

$17.5 \% \mathrm{CH}_{3} \mathrm{OH}, 13.2 \%$ methyl acetate, $42.3 \%$ propyl

Log \#660015 acetate, $27.0 \%$ toluene. Flash point $48^{\circ} \mathrm{F}$ OC.

Crystalex EPK

Kaolin

Curing Agent $\mathrm{Z}$

$50 \%$ methylene dianaline, $40 \% \mathrm{~m}$-phenylene diamine,

Log \#772598 $10 \%$ pheny glycidal ether.

CEG

Cut Aid

98\% 1,1,1-trichlorethane, $2 \%$ cutting oil.

Log \#631046

TAL

Cutscum Detergent

Mixture of nonionic surfactants, $\mathrm{pH}=7.5$.

Log \#772012

CEG 
Dairy Specialties Co., Oklahoma City, OK

Milk Stone Remover

$30 \% \mathrm{H}_{3} \mathrm{PO}_{4}+$ test for $\mathrm{Cl}+$ test for $\mathrm{PO}_{4}$.

$\mathrm{pH} \ll 0$ - strong acid; evap. to $\mathrm{H}_{3} \mathrm{PO}_{4}$.

Log \#630626

TAI

Dap, Inc., Dayton, $\mathrm{OH}$

Dap Waterless Hand Cleaner and Conditioner

$2 \%$ biodegradable sulfated aliphatic anionic

Log \#671739

detergent - 10\% lanolin. Balance - alkyl aril polyglycol ether and glycerol. No defatting solvents, no petroleum distillates.

Datnab

1,3-diamino, 2,4,6-trinitrobenzene. Probably an explosive.

Day Company

Stafoam

Urethane plastics.

Day-GIo 66

Flash point $118^{\circ} \mathrm{F}$.

Log \#661551

LWB

Dayton-Dem Kote Blue Gray 2 × 729

Solvents are ethanol, chloroform, 2 butanone

Log \#770978

and toluene. Polyester resin and propellant

CEG

is butane or propane.

Dayton Rogers Mfg. Co.

Layout Fluid Thinner \& Remover

Chiefly amyl acetate M.A.C., 200 ppm 1.1\% by volume in air.

DC SYI - KEM

Silicone, primer.

Deapa

Diethylaminopropylamine.

Deft Inc., Torrance, CA

Deft "Remove"

Contains methylene chloride, trichloropropane and methanol. Cannot be made nonpoisonous; combustible.

Delco Auto Transmission Fluid - 5 Wt. Flash point $385^{\circ} \mathrm{F}$. 
Demeron Enterprises, Inc., Louisville, KY

Vi-Lan Hand Cleanser

Log \#690516

Contains lanolin, glycerine, 2,6-dimethyl-4-hydroxylCEG chlorobenzene, water and perfume in a petroleum base (deodorized kerosene). $\mathrm{pH}$ of $1 \%$ aqueous $\operatorname{soln}=8.4$. $9.3 \% \mathrm{H}_{2} \mathrm{O}, 75 \%$ paraffin $\mathrm{HC}$ 's, $7 \%$ soap/detergent, $2 \%$ lanolin.

Deoxalum - Aluminum Cleaning Agent

$\approx 0.1 \mathrm{~N}$ in $\mathrm{H}_{2} \mathrm{SO}_{4}$ in solution with soap or detergent.

Derma San Hand Cleaner Pads

Consists of glycerine and lanolin in an aqueous

solution. Pads are made of cotton cloth.

"De-Seal" - E Paint Stripping Compound.

Solvent is alkyl chloride (3-chloropropene)

$\mathrm{CH}_{2}=\mathrm{CH}-\mathrm{CH}_{2} \mathrm{Cl}$. Sample consists of a

volatile solvent plus some gelatinous residue.

Solvent is one component chlorinated $=$ allyl

chloride or 3-chloropropene.

Deseal-2L Paint Stripping Component

Methylene chloride $\mathrm{CH}_{2} \mathrm{Cl}_{2}$. (Residue is viscus, gelatinous - not water soluble.)

$11 / 5 / 58$

$\mathrm{JE}$

Iog \#690381

Iog \#630285

TAL

Log \#630322

TAI

Diall Mesa Molding Compound

A diallyl phthalate resin, similar to Rogers molding compound.

A. B. Dick, Chicago, II

Azograph Carbon Paper

Mostly organic dyes, wax binders and carbon

black. No organic volatile solvents or

inorganic toxic materials were found.

A. B. Dick Co., Chicago, IL

Azograph Fluid

$\mathrm{CH}_{3} \mathrm{OH}+\mathrm{blue}$ dye - "\#34-2010 for use in A. B.

Dick duplicators - corrosion will result in

other duplicators."

A. B. Dick Co., Chicago, II

Azograph Fluid

Methyl alcohol

Iog \#641140

JPG

Iog \#670246

JPG, LWB

A. B. Dick Co., Chicago, II

Azograph Fluid

$\log \# 651266$

LWB

$85 \%$ methyl alcohol $+15 \%$ triethanol amine and

dye. Possibly bromthymol blue.

Log \#641522 LWB 
A. B. Dick Co., Chicago, II

Compatible Azograph Fluid

$\mathrm{CH}_{3} \mathrm{OH}+$ blue dye. (TNI $100 \% \mathrm{CH}_{3} \mathrm{OH}$ )
$\# 34-2050$.

Log \#670245

LWB

A. B. Dick Co., Chicago, IL

$\log \# 681630$

Copier 92-2175

CEG

$21.5 \%$ carbon black $+78.5 \%$ hydrocarbons

$\left(\mathrm{C}_{11}-\mathrm{C}_{14}\right)$.

A. B. Dick Co., Chicago, IL

Dispersant 92-2200

$100 \%$ hydrocarbons $\left(\mathrm{C}_{8}-\mathrm{C}_{14}\right)$.

$\log \# 681631$

CEG

A. B. Dick Co., Chicago, II

Mimeograph Ink

Boiled at $185^{\circ} \mathrm{F} 625.7 \mathrm{~mm} \mathrm{Hg}$.

A. B. Dick Co., Chicago, IL

Log \#641138

Purple Ditto Carbon Paper

Mostly organic dyes, wax binders and carbon

JPG

black. No organic volatile solvents or

inorganic toxic materials were found.

Dicup

Dicumyl peroxide.

Diesel Fuel Oil

Flash point $111^{\circ} \mathrm{F}$.

Dietzgen 200X Pencil Line Eradicator

$\mathrm{N}$-butyl alcohol - $9.86 \%$

i-propyl alcohol - $44.35 \%$

methyl alcohol - $45.79 \%$

Dietzgen 200X Pencil Line Eradicator

Volume percentage: $45 \%$ isopropanol,

45\% methanol, 10\% 1-butanol.

Dip-Seal Corp.

Dip-Seal Plastic

Polyvinyl alkyl ether resin with plasticizer $(<10 \%)$ and oil $(<5 \%)$.

Dip Seal Corp.

Dip Seal Plastic - Red \& Green

Polyvinyl butyral in castor oil base.

$7 / 26 / 62$

IL

Log \#641251

LWB

Log \#673556

JPG

$\log \# 691936-7$

LWB, FT 
Ditto Fluid

90.4\% pure methanol (spec 535A 40-65\%

$\mathrm{MeOH}, 20-40 \%$ benzene - EtOH Azeotrope).

Ditto Inc., Chicago, II

Direct Process Fluid

$\mathrm{CH}_{3} \mathrm{OH}$; "contains over $60 \%$ methanol...

contains hydroxylorganic compounds."

Fire hazard rating in class with alcohol (ethyl).

$\mathrm{ND}_{\mathrm{D}}=1.3298(18)\left(\mathrm{TNI} \mathrm{CH}_{3} \mathrm{OH}-50-60 \%+\mathrm{EtOH}\right) ;$ flash point $46^{\circ} \mathrm{F}$ OC; flammability $20-70^{\circ} \mathrm{F}$.

Diversey

Diversey DS-9-333 Stainless Steel Brightener \& Descaler $70 \mathrm{~g} / 1 \quad \mathrm{H}_{3} \mathrm{PO}_{4} ; 47 \mathrm{~g} / \mathrm{I} \mathrm{HCl} ; 10 \mathrm{~g} / 1$ alkyl phenol polyglycol ether; balance $\mathrm{H}_{2} \mathrm{O}$.

DL Skin Care Products

DL Hand Cleaner

Petroleum naphtha in a kerosene solvent base

with lanolin and hexachlorophene.

Do-AII No-Load Mounting Cement

Polyester resin with filler of chalk or kaolin.

No accelerators or catalysts detected.

Dow Chemical Co.

Chlorothene VG

Contains methyl chloroform.

Dow Chemical Co.

Dowclene EC Cleaner

$75 \%$ inhibited 1,1,1-trichloroethane $+25 \%$

tetrachloroethylene by weight. Ref: AIHA

Journal 24, No. 6541,1963 (p. 653).

Dow Corning Corp., Midland, MI

Aerospace Sealant \#92-024

Methyl silicone rubber potting resin (grey);

methyl silicone oil vehicle; grey paste, sweetish

"ketone" smell.

Dow-Corning

\#4 Compound

Methyl silicone.

Dow-Corning

200 Fluid

Dimethyl polysiloxane.
$11 / 21 / 62$

TAL

Log \#670247

LWB

Log \#673350

JPG

Log \#670217

LWB

Log \#700039

CEG

Log \#772711

$\mathrm{CEG}$

Log \#680403

JPG

Log \#660158

LWB

Log \#680641

IWB 
Dow-Corning

Stopcock Grease

Log \#660159

Methyl silicone.

LWB

Dowicide G

Fungicide; see sodium pentachlorophenate which

constitutes $75 \%$ of this material.

Dri-Glo Furniture Wax

Petroleum distillate - $100 \%$ paraffins and

Log \#641265

naphthenes; boiling point range $85^{\circ}-90^{\circ} \mathrm{C}$.

LWB

Dry Lube - Aerosel

$87 \% \mathrm{CCl}_{4}$; two very low boiling fractions - 5\% each; residue $3.6 \%$.

$12 / 11 / 62$

TAL

D. T. Cleaner

Unreacted alkali $=13.5 \mathrm{ml}$ of $1 \mathrm{M} \mathrm{H}_{2} \mathrm{SO}_{4} / \mathrm{gm}$ of

cleaner. $\mathrm{pH}$ 11.9. Offensive odor $\leq$ a pine oil

as a deodorant and germicidal agent.

DTE 24 Oil

Contains aliphatic hydrocarbon oil.

$\log \# 641296$

DuPont

Delrin (Delrin Acetal Resin)

A formaldehyde thermaplastic completely inert;

sp gr 1.4, M.P. $175^{\circ} \mathrm{C}$, nontoxic.

DuPont

Developer B

Tech. sodium sulfite; $\mathrm{pH}\left(5 \% \mathrm{H}_{2} \mathrm{O}\right.$ solution $)>11$.

Log \#680504

JPG

DuPont

Esteron 10-10

2,4-dichlorophenoxyacetic acid $53.5 \%$ acid

equivalent propylene glycol, butyl ether esters

$70.5 \%, 29.5 \%$ inert ingredients, organic ester of

2,4-D. Can cause dermatitis, avoid inhalation.

DuPont

Hylene W

$5 / 25 / 76$

Methylene bis (cyclohexylisocyanate). Strong skin irritant and sensitizer; 10 times as irritating as TDI. TLV .01C ppm; $\mathrm{mg} / \mathrm{m}^{3}$.

DuPont

Hypalon

Dupont polyethylene elastomer chunks.

DuPont

Lacquer - Duco DuPont

Log \#661552

Flash point $25^{\circ} \mathrm{F}$; sustains fire. 
DuPont

LinoWrit Rapid Processing Developer A

Tech. hydroquinone.

DuPont

LinoWrit Rapid Processing Stabilizer \#1

Tech. sodium thiosulfate; $\mathrm{pH}\left(5 \% \mathrm{H}_{2} \mathrm{O}\right.$ solution $)=5$.

DuPont

LinoWrit Rapid Processing Stabilizer \#3

DuPont

LinoWrit Stabilizer \#1 Type 200

Contains thiosulfate and sulfate anions; ammonium as cation.

DuPont

Prep Sol Solvent 3919-S

Mixture of aliphatic and aromatic hydrocarbons with some acetates.

DuPont

Thinner

Acetone $21.0 \%$, toluene $46.3 \%$, methyl cellosolve acetate (2-methoxyethyl acetate) $32.7 \%$; flash point $35^{\circ} \mathrm{F}$ OC.

DuPont

Zerlate

DuPont thiuram base fungicide.

DuPont

Zytel

A DuPont brand of nylon.

Duracryl Acrylic Lacquer Thinner

$43.5 \%$ acetone, $37.0 \%$ toluene, $5.0 \%$ cellosolve acetate, $14.5 \%$ aliphatic hydrocarbon (octane);

flash point $18^{\circ} \mathrm{F}$ oC $629.3 \mathrm{~mm} \mathrm{Hg}$.

Durakore

Liquid dental cement contains methyl methacrylate.

Dutch Boy - White Lead

Used as lubricant; $90 \%$ basic carbonate of white lead,

$10 \%$ linseed oil (manufacturer's information).
Log \#680503

JPG

Log \#680505

JPG

Log \#680506

JPG

Log \#720181

CEG

Log \#740591 CEG

Log \#680362

JPG

Log \#650842

GE, LWB 
Dutch Cleanser

$\mathrm{Cl}_{2} 35 \mathrm{ppm}$ (solutions were made up at $15 \mathrm{gms}$ per liter tap $\left.\mathrm{H}_{2} \mathrm{O}\right)$ at $25^{\circ} \mathrm{C}$ or saturated solution; $\mathrm{pH} 10.0$.

Dykem Steel Blue Low boiling hydrocarbons, petroleum ether, n-propyl acetate, blue dye; $90 \%$ ligroin and $10 \%$ propylacetate.

Dynachem Developer

Xylene.

Dynachem Resist

Petroleum naphtha + polymethyl methacrylate plastic.

Dynaloy, Inc., Newark, NJ

Uresolve Plus

75\% 2-methoxyethanol, $15 \%$ dimethylformamide, $10 \%$ diethylformamide.
Iog \#630368

TAI

$\log \# 640257$

ROM

Log \#650319

LWB

$\log \# 650318$

LWB

Iog \#680405

JPG 
Eagle Fixatif Aerosol

55\% Ethanol

$35 \%$ freon propellants (18\% B.P. $11^{\circ} \mathrm{C}$,

$15 \% \approx-10^{\circ} \mathrm{C}, 2 \%-25^{\circ} \mathrm{C}$ )

$8 \%$ polyvinyl acetate

$2 \%$ ethyl acetate

Eagle Fixatif Art spray Matte Finish

$81.4 \%$ ethyl alcohol, $11.2 \%$ methyl alcohol, $6.4 \%$ methylene chloride, $<1 \%$ ethyl acetate. Contains a freon as propellant.

Eagle Fixative

Methyl alcohol, isopropyl alcohol, and a chlorinated hydrocarbon

Eagle-Picher Floor Dry 85

Less than $0.5 \%$ "free" silicon present. Contains $\mathrm{Al}, \mathrm{Mg}, \mathrm{Na}, \mathrm{Fe}, \mathrm{Ca}$ as cations. Silicate as anions. No carbonates.

Eastman Kodak

$\mathrm{X}$-Ray Fixer \& Replenisher

Fixer - $10 \% \mathrm{Na}_{2} \mathrm{~S}_{2} \mathrm{O}_{3}, 5 \% \mathrm{H}_{2} \mathrm{SO}_{4}$ in $\mathrm{H}_{2} \mathrm{O}$

Replenisher - $20 \% \mathrm{H}_{2} \mathrm{SO}_{4}$, ${ }_{20 \%} \mathrm{Al}_{2}\left(\mathrm{SO}_{4}\right)$ in $\mathrm{H}_{2} \mathrm{O}$

Eastman Kodak

Eastman 910 Adhesive

Methyl - 2 - Cyano acrylate

Eastman Kodak

Kodak Autopositive Resist Type 3 MX740-1

Inorganic - Na. Solvent - ethylene glycol monomethyl ether acetate. Plastic - polyacrylamide and isoprene copolymer.

Eastman Kodak

Eastman "910" Catalyst

Phenylethylethanolamine

Eastman Kodak

Kodak Developer Iinograph 1000 Iiquid Processing Kit Contains sodium sulfite, hydroquinone, sodium hydroxide, sodium carbonate, $\mathrm{KBr}, \mathrm{KCl}$ and water
Log \#670424

JPG

$10 / 13 / 70$

CEG

Log \#620790

PG

Iog \#713566 \& 713560 CEG/WLC

Log \#661492

JPG

$2 / 1 / 71$

CEG

Log \#710198

CEG 
Eastman Kodak Co.

Kodak Film Cleaner

15\% Heptane; remainder as chlorinated hydrocarbons $\left(43-60^{\circ} \mathrm{C}\right)$; one being ethyl chloride

Eastman Kodak

Kodak Metal-Clad Plate Etchant Contains $\mathrm{Fe}$ and $\mathrm{Cr}$ and $\mathrm{H}_{2} \mathrm{SO}_{4}$

Eastman Kodak Co.

Kodak Metal Etch Resist

Inorganic - $\mathrm{Na}, \mathrm{Cu}, \mathrm{Mg}, \mathrm{Pb}, \mathrm{Fe}, \mathrm{Min}$;

Solvent - xylene; Plastic-polyisoprene

Eastman Kodak

Kodak MX540-KTFR

Inorganic Zr, Na; Solvent - xylene;

Plastic - Isoprene

Eastman Kodak

Kodak Photoflo 200

Small amount of ethylene glycol in water

with wetting agent and some sulfates

Eastman Kodak

Kodak Photoresist Type 2

No inorganic

Solvent - monochlorobenzene

Plastic - polyacrylamide

Eastman Kodak

Kodak Stabilizer Iinograph 1000 liquid processing kit Contains sodium thiosulfate, sodium sulfite, formalin, sodium carbonate

Eastman Kodak

Kodak Stabilizer 2 linograph 1000 liquid processing kit Contains sodium thiosulfate, sodium sulfite, formalin, sodium carbonate

Eastman Kodak

Kodak Part A Stop Bath Iinograph 1000

Iiquid Processing Kit

Contains sodium sulfate, water, alkali
$3 / 16 / 62$

JE

Iog \#710733

CEG

Iog \#702383

CEG

Iog \#702384

Log \#713377

CEG

Iog \#702383

CEG

Log \#710199

CEG

Log \#710200

CEG

Iog \#710196

CEG 
Eastman Kodak

Kodak Part B Stop Bath Linograph 1000

Liquid Processing Kit

Contains acetic acid

Eastman Kodak

Kodak Rapid Fix

Sodium thiosulfate

Eastman Kodak

Kodak X-Ray Developer

(Developer) Sol'n A $7.1 \mathrm{~N}$ OH- Hydroquinone

Sol'n B (replenisher) $8.9 \mathrm{NH}+50 \%$ acetic acid

Easy Flo

Silver Solder

Wire $1 / 32-30.1 \% \mathrm{Cd}$
Sheet $-21.0 \% \mathrm{Cd}$ is not known for these

Ecco Brand

Ecco Bowl Cleaner \& Scale Solvent

Approx. 40\%-50\% $\mathrm{HCl}$; water, dye

Eccospheres

Inorganic glass $77 \% \mathrm{SiO}_{2}$

Edmund Chemical Co., Albuq., NM

Ecco Brand. Ice Melter

$97.7 \% \mathrm{KCl} ; 0.585 \% \mathrm{NaCl} ; 0.003 \% \mathrm{CaCl}_{2}$;

$0.012 \% \mathrm{MgCl}_{2} ; 0.513 \% \mathrm{H}_{2} \mathrm{O}$ (Ioss $@ 600^{\circ} \mathrm{C}$ )

EFFA Spray Duster

Freon with a trace of hydrocarbon oil -

possibly from the can coating

Electroglo Co., Chicago, II

Electroglo 200

$53 \%$ conc. $\mathrm{H}_{3} \mathrm{PO}_{4} ; 27.0 \%$ tri-n-buty 1 phosphate; $26.6 \%$ n-butanol

"Electroless" Black Magic for Aluminum

Mo $1.9 \%$ in can as $\left(\mathrm{NH}_{4}\right)_{2} \mathrm{MOO}_{4}$;

B $4.8 \%$ in can as $\mathrm{H}_{3} \mathrm{BO}_{3} ; \mathrm{Si}<0.5 \%$;

$\mathrm{Na}<0.5 \% ; \mathrm{CoSO}_{4} 0.7^{3} \mathrm{H}_{2}{ }^{3}$ remainder with
Log \#710197

CEG

Log \#661429-30

JPG

Iog \#660513-14

JPG

Log \#620871

$P G$

Iog \#630407

$\mathrm{RM}$

Log \#680036

JPG

Log \#680821

LWB

Log \#680407

JPG

Log \#641736

JPG 
EImox $G-20$

$\mathrm{Na}, \mathrm{B}$ only elements detected $\mathrm{PH}=9$

Emerson Cummings, Gardenia, CA

ECCO-Strip 93

85-90\% methylene chloride $0.5 \%$;

$\mathrm{CCl}_{4}+\mathrm{CHCl}_{3}+$ methylchloroform;

9-10\% formic acid

Engelhard

Silver Solder

$\# 6-355 \quad 0.1 \%$ ca

$\# 50 \quad 17.8 \% \mathrm{Cd}$ (TNI 18\%)

Englehard-355 Silver Solder $.045 \%$ ca.

EPD-TC-438

Epoxy scaling kit, polyamide

EPD-TC-459

Epoxy molding kit, polyamide

Epocast Versamid Catalyst

A polyamide catalyst

Epo-Iux 100 Coating E-I White

Contains toluene and GME (ethylene glycol

monoethyl ether). No lead.

Epon R Shell

Epoxy composed of diphenylpropane.

Amine hardener composed of p-phenylenediamine

and 2,3,5 tri - (dimethylamino) - methyl phenol

Epolux Thinner

Contains ethylene glycol monoethyl ether and

a mixture of xylenes.

Epoxy Plastics

Plastics based on resins made by reaction of epoxides or oxiranes with other materials such as amines, alcohols, phenols, carboxylic acids, acids anhydrides and unsaturated compounds. Dermatitis attributed to the amines used as curing agents.
Iog \#762662

CEG

Log \#660511-12

JPG

Log \#712548

CEG

Log \#713966

CES

Log \#761106

CEG 
Epoxy Resin for Curing Agent $Z$

Log \#772599

Contains bis phenol A; Epichlorohydrin

Epy

Epoxy

Ethyl Centralite

Diethyldiphenyl urea

Ethyl Selenac

Selenium diethyldithio-carbamate

Ethyl Tuad

Tetraethylthiram disulfide accelerator

Exxon Corp.

Caloria HT-43

Iog \#773928

Contains aliphatic hydrocarbons 
Flectol-H

Antioxidant, amino compound

Flexzone $\mathrm{C}-\mathrm{H}$

$\mathrm{N}$-phenyl-N-cyclohexyl-p-phenylaminediamine

Flubber

Polybutadiene in mineral oil prepared by action of sodium on 1,3 - butadiene; a synthetic rubber product

Fluro-Bond \& Fluoro-Etch

Mixture of sodium naphthalate and tetrahydrofuran

Flux for Stainless Steel

Flux contains iron, nickel, manganese,

Log \#650271 silica and boron

Foresight, Inc. - Denver, CO

F101 Foresight Cleaner

$\mathrm{Na}$ and Si only elements detected. Positive

GE

test for carbonate and silicates. $\mathrm{PH}=9$

Formica Solvent

Isopropyl ether, ethyl acetate, several

low boiling aliphatic esters, alcohol, ketone, ether compounds and an aromatic component

Formica Solvent

Isopropyl ether, ethyl acetate, several low boiling aliphatic esters, alcohol, ketone, ether compounds and an aromatic component. 9 components total

Foto Foil Co.

Foto Foil Etching Powder

$0.01 \% \mathrm{Soln}=2.5 \mathrm{ppm} \mathrm{Na}$

Iog \#630325

TAI

Log \#762658

CEG

Log \#692902

EG

Franklin Formula \#1010 Floor Wax

Carnuba wax with an amine emulsifier in

Log $\# 740502$

an aqueous solution

Franklin Pink Velvet Hand Soap

$70 \%$ boron (probably as borox) $<0.5 \%$ pine-0il

Log \#701083

perfume. 3-4\% lanolin. Remaining material

CEG

is sodium \& potassium soap. Trace of

hexachlorophene 
Fernox F-II Rust Inhibitor

$\mathrm{Na}, \mathrm{B}$ only elements detected $\mathrm{pH}=9$

Log \#762663

CEG

Fibrene $\mathrm{C}-400$

Talcum, filler for potting materials

Filter Pad Coating Oil

This is a petroleum base product, soluble

in toluene, has density of $760 \mathrm{mgms} / \mathrm{ml}$.

Is adaptable to fluorimetric analysis

with a lower limit of sensitivity of $20 \mathrm{mgms}$.

Suggest sampling in toluene or millipore filters.

Finch Paint Thinner TI-26

49.5\% ethyl acetate; $27.4 \%$ hexane;

Log \#713568

$23.2 \%$ xylene; no alcohols or halogenated

CEG

hydrocarbons

Finch Paint Thinner TL-29

$6.6 \%$ ethanol; $51.6 \%$ heptane; $10.4 \%$ ethyl

Log \#713569

acetate; $31.5 \%$ hexane; no aromatics or

halogenated hydrocarbons

Finch Paint Thinner TL-52

40.5\% hexane or heptane; $7.5 \%$ ethanol;

Log \#7I3567

$35.8 \%$ ethyl acetate; $13.5 \%$ xylene;

CEG

2.9\% methanol; no halogenated hydrocarbons

Fishers stain-Off

The cleaner is diethylene glycolmonoethyl

Log \#661675 ether in an unsaturated fatty acid based

oil. Infrared, fractional distillation

Fisher "Stain-Off"

Diethylene glycol monoethyl ether in

an unsaturated fatty acid-based oil

Flamemaster Co.

Flamemastic \#71A Fire Protection Coating

Solvent is water. Glass fibers present.

CEG

P, Si, B, Mg, Ti, Na, Al, Ca, Fe present

Log \#661675

JPG/LWB

Iog \#772987

$\mathrm{CEG}$

Flamemaster Fire Protection Coating \#77

Contains $\mathrm{B}, \mathrm{Si}, \mathrm{Al}, \mathrm{Sb}, \mathrm{Mg}, \mathrm{Fe}, \mathrm{Ca}$,

Log \#774089

$\mathrm{Na}, \mathrm{Ti}$

WE

Flamemastic \& Flamemastic Thinner Toluene

Log \#630032

TAI 
Freon TA

Freon TA; $20 \%$ acetone to $80 \%$ of 2 nd phase,

Iog \#630105

presumed to be Freon TF

Fry Plastics Plexiglass \& Lucite Cement $100 \% 1,2$ - dichloroethane

Log \#690259

CEG

Furan Plastics

Plastics based on resins in which the furan ring is an integral part of the polymer chain. Made by polymerization of furfural, furfural alcohol, or other furan-ring compounds.

Furnex

Carbon black grains

Fusel Oil

Isoamyl alcohol.

Fusion Silver Brazing Paste \#S-4-1050

Iog \#660533

7.5\% cadmium

JPG 
GAF Corp.

Microline-Positive Working Photoresist PRIO2

Log \#702387

No inorganics. Solvent - ethylene glycol monomethyl

CEG

ether acetate. Plastic - polyacrylamide and

isoprene copolymer.

Galex

Rosin

Garnet Abrasive

$<10 \%$ quartz

Iog \#771194

CEG

Gates Engineering Co.

GACO Neoprene Thinner

100\% 2-butanone; TLV $200 \mathrm{ppm} ; \mathrm{mg} / \mathrm{m}^{3}$.

Log \#661834

LWB

G. C. Electronics Co.

G. C. Acrylic Cement \#402

Flash point $<41^{\circ} \mathrm{F}$ TCC

G. C. Electronics Co., Los Angeles, CA

G. C. Cement Thinner \#28-2

$3.1 \%$ methyl alcohol, $24.7 \%$ acetone, $23.7 \%$ ethyl

Log \#680039 acetate, $22.7 \%$ toluene, $25.8 \%$ n-butyl acetate.

G. C. Electronics Co.

G. C. Lacquer Thinner

Flash point $<40^{\circ} \mathrm{F}$ TCC, DC.

G. C. Electronics Co., Rockford, II

Spra-Kleen \#8666-16

Electrical contact cleaner and lubricate. Contains

Log \#730859 $\approx 95 \%$ methyl chloroform, $\approx 5 \%$ methylene chloride. Propellant is Freon 12.

G. C. Electronics Co.

Log \#651101

Strip-X

Methyl chloride $30 \%$ plus ammonia and phenol.

LWB

Gelvatol

Water soluble polyvinyl alcohol resin. White powder, decomposes above $200^{\circ} \mathrm{C}$, flash point greater than $392^{\circ} \mathrm{F}$.

General Cement Co.

Radio Service Solvent

$32 \%$ acetone, $37 \%$ ethyl acetate, $29 \%$ aromatic

LWB

solvent carrier, $2 \%$ unidentified trace hydrocarbons.

General Cement Co.

Radio-TV Cement Solvent

$\mathrm{CEG}$

Contains ethyl acetate, amyl acetate, butyl

acetate, acetone; no toluene detected.

Log \#761097

$10 / 10 / 61$

$\mathrm{JE}$ 
Genetron

Trifluorotrichloroethane, same as Freon 113,

halogenated hydrocarbon refrigerant.

Glastic

Glass-reinforced polyester plastic molding compound.

Glaze Remover

Contains chloroform/xylene.

Log \#772608

WE, CEG

Glyptals (glyptol)

Resins made from phthalic anhydride and glycerol.

Go-Jo Waterless Handcleaner

Deodorized kerosene plus lanolin and silicone

Log \#661576

LWB

B. F. Goodrich

Adhesive A $1178 \mathrm{~B}$

Mixture of phthalic acids and aliphatic dicarboxylic

Log \#701644

IWB, CEG

acids. Solvent is methyl isobutyl ketone.

B. F. Goodrich

Adhesive A $1178 \mathrm{~B}$

Solvent is methyl ethyl ketone.

Log \#730137

CEG

B. F. Goodrich

Flexseal \#l Flashing Adhesive CAI056 White

Solvent is TCE, adhesive is a latex rubber or butyl

Log \#771443

$C E G$ rubber.

Granodine Compound

A zinc phosphate surface treatment compound.

Granodraw Compound

A phosphate surface treatment compound.

Grease-OL

Flash point $110-115^{\circ} \mathrm{F}$

Green's Sanitary Supply

Allsheen

Solvent phase consists of unsaturated petroleum

distillates. B.P. range $157-192^{\circ} \mathrm{C}$ at $760 \mathrm{~mm} \mathrm{Hg}$;

miscible $\overline{\mathrm{W}}$ Velva Sheen; flash point $126^{\circ} \mathrm{F}$.

Green's Sanitary Supply

Luron

2.35\% lanolin, major borax, trace silicates, good spectral purity, $\mathrm{pH}$ of $1 \%$ slurry - 9.1, $\%$ insol. in $1 \%$ slurry $-0.25 \%$. Odor - soapy, mild; particle size uniformity - good; dust generation when poured - none.

$9 / 12 / 62$

IL

Log \#672355

JPG 
Green's Sanitary Specialties, Albuquerque, IM

$P$ \& $\mathrm{E}$ Cleaner

$3.6 \% \mathrm{HCl}, 7.2 \%$ oxalic acid, $88 \%$ water, trace deordorant and detergent.

Green's Sanitary Specialties

$P$ \& $E$ Cleaner

Acid $=8 \%$ as $\mathrm{HCl}, 91 \%$ water, $1 \%$ cleaners and detergents.

Log \#661646

JPG

Log \#721255

CEG

GSA

Mop Dressing Oil

Flash point $176^{\circ} \mathrm{F}$ OC. GSA Stock No. 704169 .

Log \#660427

$\mathrm{GE}$

Log \#630367

GSA

TAL

Chlorine (ppm) approx. I; solution made up at

$15 \mathrm{gms}$ per liter tap $\mathrm{H}_{2} \mathrm{O}$ at $25^{\circ} \mathrm{C}$ or saturated solution. $\mathrm{pH} 10.5$ (compared with Dutch Cleanser $\mathrm{pH}=10.0, \mathrm{Cl}_{2}=35 \mathrm{ppm} . \quad$ GSA \#7930-663-9257.

GSA

Log \#660051

Scouring Powder

$\mathrm{pH}=7.0$, chlorine $=$ N.D. Stock No. 7930-205-

0442 - Type II without bleaching, Fed. Spec. P-S-311c.

LWB

GSA

35X Thinner

$15.9 \%$ isopropanol, $18.4 \%$ acetone, $12.7 \%$ n-hexane,

Log \#680360

JPG

$12.3 \% \mathrm{MIBK}, 40.7 \%$ toluene; flash point $32^{\circ} \mathrm{F}$ OC.

Gulf Microwax \#75

Tungsten dispersal in microcrystalline wax.

Petroleum wax - high molecular weight aliphatic

hydrocarbon.

Gunk Laboratories

Carbon Met

$20 \%$ dichloromethane (methylene chloride) in

petroleum distillate, similar to benzene. Boiling

point $130^{\circ} \mathrm{C}$, similar to benzene.

Log \#680501

IWB

$8 / 7 / 61$

$\mathrm{AJE}$ 
Habcool \#318

A petroleum based oil containing sulfur

$\log \# 650097$

(contains $6 \%$ sulfur and $2.9 \%$ chlorine).

IWB

Hale Co.

Pepco-415-Antiseptic Hand Cleaner

$0.45 \%$ lanolin, major borax, minor silicates, fair spectral purity, $\mathrm{pH}$ of $1 \%$ solution - 9.3, $\%$ insol. in $1 \%$ solution - $0 \%$. Odor - soapy, strong; particle size uniformity - fair; dust generation when poured - much.

Hale's Satin Finish (Silicone Polish)

Wax and silicone suspended in a $1: 1$ mixture of isopropanol and petroleum ether. The solvent odor is masked by the strong perfume which is present.

Handy \& Harman

Handy Flux

Allergic individuals may suffer skin irritation.

When overheated, the fumes are irritating to the eyes, nose and throat.

Hanley's Coroban Poly Epoxy, Part A

Contains toluene and ethylene glycol monoethyl

ether as solvents; no lead. Flash point $24.5^{\circ} \mathrm{C}$.

Iog \#672639

JPG

Log \#641093

LWB

Log \#713965

CEG

Hanley's Coroban Poly Epoxy, Catalyst B

Contains toluene and ethylene glycol monoethyl ether as solvents; no lead. Flash point $23^{\circ} \mathrm{C}$.

Log \#713964 $\mathrm{CEG}$

Harflex 150

High molecular weight ester.

Heliozone

Petroleum wax.

Herculene Erasing Fluid K\&E 580260

50\% Freon 11, 10\% Freon 21, 40\% isopropy1

Log \#620567 alcohol; nonflammable by $O C$.

Hermes Bond 730

Methyl ethyl ketone and 2-5\% acetone.

Log \#630408

ROM, GC

Hermes Cement

Methyl ethyl ketone.

Log \#630408

Hermes II Oil

Log \#680770

High purity mineral oil.

LWB 
Hersen Chemical, Detroit, MI

\section{Rance-Rid}

Sodium perborate, $\mathrm{NaBO}_{3} \times 4 \mathrm{H}_{2} \mathrm{O}\left(\mathrm{NaBO}_{2} \cdot \mathrm{H}_{2} \mathrm{O} \cdot 3 \mathrm{H}_{2} \mathrm{O}\right)$, traces of calcium, magnesium (spectrograpn, atomic absorption).

Hexaver

Disodium dihydrogen cyclohexane diamine tetraacetate.

Heyer, Inc., Chicago, IL Spirit Fluid

$\mathrm{CH}_{3} \mathrm{OH}$, "contains methanol;" \#475 "a superior fluid for use on all direct process duplicators." $N_{D}=1.328(18) ; T L V ~ 200 \mathrm{ppm}, \mathrm{mg} / \mathrm{m}^{3} ;$ flash point $50^{\circ} \mathrm{F}$ OC; flammability $20^{\circ}-70^{\circ} \mathrm{F}$.

Hi Grade Alloy Corp.

Lacquer Thinner SM20O

$100 \%$ toluene

Log \#670250

LWB

E. V. Hill, Inc.

White Smoke Candle \#10-030

Residue is $\mathrm{ZnCl}_{2}+\mathrm{C}$; telecon with supplier

(Ditto) $8 / 31 / 70^{2}$ TLV $1.0 \mathrm{mg} / \mathrm{m}^{3}-\mathrm{ZnCl}_{2}$.

Log \#661758

HiTri

Trichloroethylene.

Hochwald Company, San Francisco, CA

Dust Away Mop Dressing

Mineral oil carrier for a wax.

$\log \# 641264$

IWB

Hockwald Company

"H" Nu-Clean

8. $1 \% \mathrm{HCl}$, no oxalic acid, $10 \%$ alkyl aryl

$8 / 31 / 70$

LWB polyglycol ether, trace deodorant, balance is water.

Hoppe's Powder Solvent Nitro \#9 $1.61 \%$ nitrobenzene, $2.40 \% \mathrm{NH}_{3}, 15.5 \%$ ethanol, approximately 5\% amyl acetate, approximately JPG

Log \#680052 JPG $75 \%$ petroleum distillates. Boiling point $125^{\circ} \mathrm{C}$ and greater. 
Houghto-Clean 224 Paint stripper

$70 \%$ methylene chloride, $30 \%$ commercial

Iog \#661263

cresols; flash point - none; boiling point

$90^{\circ} \mathrm{F}$.

E. F. Houghton \& Co.

Cindol 3102

Less than $10 \%$ fatty acid esters and $91 \%$

minimum of petroleum oil. Does not contain

chlorine or sulphur.

E. F. Houghton \& Co.

Cutmax \#50 Cutting Oil

Log \#660548

Sulphur, chlorinated active cutting oil; dilution

$1: I$ in light machine oil; flash point $283^{\circ} \mathrm{F}$ OC.

E. F. Houghton \& Co.

Cutmax Cutting Oil \#570

Petroleum based oil containing sulfur in

relatively large quantity. New oil \% by wt.

chlorine $=0.89$; used oil \% by wt. chlorine

$=0.44$.

HR 100 Photoresist

Contains mixed xylenes. $\mathrm{C} \equiv \mathrm{N}$ group present.

Log \#774183

WE

HR 100 Photoresist Developer

Log \#774188

Contains mixed xylenes.

WE

Hunt Waycoat IC Resist

No inorganics. Solvent - xylene; plastic -

Log \#702386 polyisoprene.

$\mathrm{CEG}$

Hydra-Clene

Wetting agent.

Hydropol

Combustible hydrocarbon.

Hyvac Oil

Flash point above $230^{\circ} \mathrm{F}$ OC.

Hyvar X - Hydrasol X

Hyvar X - wettable powder containing $80 \%$ bromacil

[bromacil - 5-bromo - 3-sec. butyl - 5-methyluraci]

$\log \# 651417$

(a herbicide)]. Hyvar X-WS - water soluble powder with $50 \%$ bromacil.

JPG 
Ice MeIters

Major constituent $\mathrm{CaCl}_{2}$; traces of $\mathrm{NaCl}, \mathrm{LiCl}$,

Log \#650808 $\mathrm{MgCl}_{2}, \mathrm{AlCl}_{3}$.

Incusil \#10

$<0.05 \%$ cadmium

Log \#661525

Incusil \#15 Solder

\% cadmium.

Indalloy \#1I solder

$96 \%$ lead, $4 \%$ indium (TNI 5\% In $+95 \% \mathrm{~Pb}$ ).

Log \#660573

Log \#650342

LWB

Indco Lab Corp., Santa Monica, CA

Log \#673124

Indco 217 Hand Cleaner

$35.65 \% \mathrm{H}_{2} \mathrm{O}, 38.75 \%$ 5-m-propyl nonane, $7.60 \%$ fatty

JPG acid diethanolamide (nonionic surf. act. agt.), $12 \%$ nonylphenol ethylene oxide (nonionic surf. act. agt.), $6 \%$ sodium polyphosphate. Hexachlorophene not detected.

Indco Lab Corp., Santa Monica, CA

Log \#701765

Indco 217 Cream Soap Hand Cleaner

CEG

$14.2 \% \mathrm{H}_{2} \mathrm{O}, \sim 71 \%$ paraffin hydrocarbons, $\sim 3 \%$

lanolin, $\sim 5 \%$ soap and detergents, $<5 \%$ triethanolamine,

$<1 \%$ perfume. $\mathrm{pH}$ of $1 \%$ solution $=8.35$.

Indust-Ri-Chem Lab, Richards on, TX

Log \#774093

J-44 Strip Solvent

CEG

Contains acetone, ethylacetate, methylene chloride, and phenol.

Indust-Ri-Chem Lab, Richards on, TX

Resist Strip-J-100

$40 \%$ perchloroethylene, $20 \%$ orthochlorophenol,

$40 \%$ phenol.

Interior Enamel Paint White KM8410

Pigment contains 56.7\% $\mathrm{TiO}_{2} ; 5.2 \% \mathrm{SiO}_{2} ; 38.1 \%$

$\log \# 751447$

$\mathrm{CaCO}_{3}$. Vehicle contains $45.6 \%$ soya alkyd;

$54.3 \%$ thinner.

International Paints Itd. of Canada

Intermastic Intumescent Fire Retardant \#c68-777

Log \#670185

JPG

Solvent is $\mathrm{H}_{2} \mathrm{O}$ and alcohol; $\mathrm{P}, \mathrm{Si}, \mathrm{Mg}, \mathrm{Ti}$,

Log \#772596

$\mathrm{Na}, \mathrm{A}], \mathrm{Ca}, \mathrm{Fe}$ present.

Interstate Business Machine Co. (International)

Facsimile Posting Fluid.

$81 \%$ acetone, $19 \%$ methyl alcohol. 
JeweI

Jewel Met-All Aluminum Polish

Major components: Fine grit alundum $\left(\mathrm{Al}_{2} \mathrm{O}_{3}\right)$,

Log \#760201

rouge $\left(\mathrm{Fe}_{2} \mathrm{O}_{3}\right)$, silica $\left(\mathrm{SiO}_{2}\right)$ suspended in $\mathrm{a}$

JPG

paste of waxes and ammonium oxalate $\left(\mathrm{NH}_{4}\right)_{2} \mathrm{C}_{2} \mathrm{O}_{4}$.

Spectrograph, petrographic microscope, wet

inorganic.

Johns-Manville

$2 / 73$

Maxinite \#36

Manufacturer

$30 \%$ asbestos.

$5 / 5 / 77$

Johns-Manville

Manufacturer

Natural uncalcined diatomaceous earth, amorphous silica. Fine fluffy powder, off-white color; M.P. $1400^{\circ} \mathrm{C}$; conversion to cristobalize begins (a) $450^{\circ} \mathrm{C}$; if $\mathrm{NaCl}$ \& Ca carbonate present, conversion is complete @ $800^{\circ} \mathrm{C}$ (ILO Dictionary). TLV $1.5 \mathrm{mg} / \mathrm{m}^{3}$ respirable dust; 1976 addition to notices of intended changes. Decomposition products: Unknown, probably none. Calcining heating below m.p., without flux, will produce product wath 5 to $10 \%$ cristobalite. Flux calcining $\rightarrow 35-45 \%$ cristobalite. Used as insulation or filtration media.

Johnson's Wax Water Soluble Cutting Fluid THI3I Contains mitrites and amines.

$C E G$

Jointers Cement

$<5 \%$ asbestos by microscope examination.

Log \#722724

Emission spect. shows $\mathrm{Fe}, \mathrm{Mg}, \mathrm{Si}$ as major

$\mathrm{CEG}$ components. 
Kadox

Zinc oxide.

Kano Molyfilm

Contains molybdenum di-sulfide; nonflammable in

Log \#714179 open flame at 6" and 12".

Kee Chem 56 Degreaser

$39.2 \%$ total alkali as $\mathrm{Na}_{2} \mathrm{O}, 16.3 \% \mathrm{NaOH}, 23.7 \%$

Log \#691401

carbonates $\left(\mathrm{Na}_{2} \mathrm{CO}_{3}\right)$, balance is poly phosphates,

CEG soap, resins and wetting agents.

Kel-F 81 Plastic

Replacement for Kel-F Plastics 270 and 300 of the same chemical identity. A chlorotrifluoroethylene (-CF ${ }_{2} \mathrm{CCFCl}$-) thermoplastic resin of approximately $1300^{2}$ to 1600 molecular weight. Melting point $414^{\circ} \mathrm{F}$; chemically inert after cure and has high thermal resistivity. Available in the following chemical forms: Kel-F8I Plastic, KF-6050, KF-605I, KF-6060, KF-606I, Ke1-F81 Plastic with 25\% plasticizer, KF-6052.

Kelvar

Polyamide with amine catalyst; contains $\mathrm{Na}, \mathrm{Si}$,

Log \#770104 $\mathrm{B}, \mathrm{Fe}, \mathrm{Mg}$.

Kem Kool

Approximately $75 \%$ water, sodium carbonate, $\mathrm{CEG}$ ethylene glycol, organic dye.

Kem Tex Plastic

Plastic is a phenolic/formaldehyde resin with

Log \#681829

a self-hardening "resole" resin. Filler is wood

CEG

flour. Plasticizer is tri-B-chloroethyl phosphate and the filler is wood flour.

Kenna Metal

Niobium pentoxide.

Kleer-Flo Co., New York, NY

Hi-T Degreasol

Petroleum distillate of 7 components; primarily

aliphatic hydrocarbons with small amount of coloring

agent which may contain some aromatic compound.

Boiling range $155-165^{\circ} \mathrm{C}$; flash point $109^{\circ} \mathrm{F} \mathrm{CC}$.

TNI \#4801; high boiling naphthas, no chlorinated

solvents, less than $0.5 \%$ detergent, perfuming agent.

Kleen Sweep Ice Remover

Chlorides of $\mathrm{Ca}, \mathrm{Al}, \mathrm{Ba}, \mathrm{Na} ; 15 \% \mathrm{Ba}, 13 \% \mathrm{Na}, 70 \%$

Log \#630265

TAL

$\mathrm{Ca}, 2 \% \mathrm{Al}$. 
KIeen Sweep Ice Remover

$32.1 \% \mathrm{Ca}, 64.1 \% \mathrm{Na}, 2.5 \% \mathrm{~K}$; as chloride and

Log \#700303

hydroxide $-83.4 \%$ chloride and $16.6 \%$ hydroxide.

CEG

(See additional information attached to Product

File Card.)

Kmer

$\mathrm{Xylene}$ and polyacrylonitrile plastic.

Log \#650316

LWB

Knadjians Magic Handy Dry Spotter

Methyl chloroform.

Log \#721014

LWB

Knadjians Magic Handy Dry Spotter Contains 1,1,1-trichloroethane.

Log \#760697 CEG

Knadjians

Picrin Dry Spotter

$60 \%$ 1,2-dichloropropane, $40 \%$ methylchloroform.

Log \#670879

LWB

Koh-I-Lar Liquid Eraser

Contains soap and sodium hexametaphosphate

$8 / 15 / 62$

in water.

Koh-I-Noor Rapidograph Inc., Bloomsbury, NJ

Koh-I-Noor Drafting Film Iiquid Eraser 291-F

Aqueous solution, $\mathrm{pH}=8$.

LL

Log \#780414

CEG

Kool Kleen

No qual. performed; strong ammonia odor;

Log \#702411 $\mathrm{pH}=9.4 ;$ ammonia smell. When heated to boiling, an ammonia smell occurred. Cut 30 to 1 with water - $\mathrm{pH}=9.0$; no ammonia smell.

Kool Kleen

Aqueous solution, $\mathrm{H}_{2} \mathrm{O}$ distills leaving colored residue, $\mathrm{pH}-9.5$ solution, 8.7 distillate, 9.5 residue.

Kool Kleen Machine Coolant

Concentrate contains 0.33 gms of soluble oil per CEG ml. Dark green; $\mathrm{pH}$ - dilute (80-1) 9.0; concentrate

Log \#650265 10.2

Kosmos

Carbon black.

$\mathrm{KPR}-1$

Log \#650313

Methylcellosolve acetate + styrene based plastic.

LWB 
$\mathrm{KPR}-2$

Log \#650314

Chloro benzene + styrene based plastic; sensitive LWB to light.

Log \#650323

KPR Developer

LWB

Kralac

Styrene resin grains.

Krylon Crystal Clear

Contains $20 \%$ methylene chloride; $80 \%$ toluene.

Krylon Crystal Clear \#1303

$43 \%$ methylene chloride, $51 \%$ toluene $+6 \%$

polymethylmethacrylate. Flammability -

Log \#651403

\#680816

$>200^{\circ} \mathrm{F}$; spray is combustible, but non-

LWB

flammable.

Krylon Workable Fixalif \#1306

Solvents are methanol and acetone. No aromatics.

Log \#761748

CEG

KTFR

Petroleum naphtha + polyacrylonitrile plastic.

Log \#650315

LWB

KTFR + KMER Developer

Petroleum naphtha

Log \#650317

LWB

$\mathrm{K} \& \mathrm{~W}$ Hand Cleaner (Waterless Hand Soap)

Partially deodorized kerosene with $\mathrm{NH}_{4} \mathrm{OH}$

$\log \# 671578$

and lanolin. $\mathrm{pH}=8.6$.

Kyanite

Aluminum silicate mineral $\mathrm{Al}_{2} \mathrm{O}_{3} \mathrm{SiO}_{2} \cdot$ 
Lacquer Thinner

Several petroleum distillates resembling

kerosene and petroleum naphtha, first

distillate at $130-140^{\circ} \mathrm{C}$; flash point

$25^{\circ} \mathrm{C}$.

Lake Welding Products, Inc., Muskegon, MI

D-Spat

Heavyweight aliphatic hydrocarbon oil with

Log \#730006

CEG

a mixture of $\mathrm{CO}_{2}$ and nitrous oxide as propellants.

Lava

Approximately 5\% quartz, 10\% amorphous

Log \#672188 silica, balance silicates.

Leak Detector - Leak Tec

Aqueous detergents. Leak Tec \#277C - pH 4.6,

JPG

Leak Tec \#277NE - pH 7.4. Slightly acidic

Iiquid.

Leak Tec 277-C

Slightly acidic liquid detergent.

Log \#641541

LWB

Lectra NIK

Sel-Rex nickel plating solution.

Lexso1 408

Flash point $92^{\circ} \mathrm{F} \mathrm{CC}$.

Iino-Kleener Linoleum Cleaner

Nontoxic, a liquid detergent.

Log \#641338

LWB

Liquid Paper Corp., Dallas, TX

Liquid Paper Thinner 565

Log \#780412 $\mathrm{CEG}$

Contains 1,1,1-trichloroethane and

trichloroethylene.

Lithoform \#32

Zine phosphate.

Lithographic Blanket Roller Wash

$20 \%$ methylene chloride, $80 \%$ petroleum

Log \#650778

naphtha; flash point $106^{\circ} \mathrm{F} 628.2 \mathrm{~mm} \mathrm{Hg}$.

LWB

Litho Plate Solution

$\mathrm{H}_{3} \mathrm{PO}_{4}$ in $\mathrm{H}_{2} \mathrm{O}$ solution $+\mathrm{NH}_{3}+\mathrm{H}_{2} \mathrm{SO}_{4}$ (minor

Log \#660343 components). 
Lix Corp., Kansas City, MO

IIX

$50 \% 1,1, I$-trichloroethane, $50 \%$ petroleum

$\log \# 630762$ distillate of kerosene range.

Lix Corp., Kansas City, MO

Lix Electrical Cleaner

TNI \#1989 - methyl chloroform 32.7\%, perchloroethylene $15.3 \%$, nonaromatic saturated HC $52.0 \%$ - (1952).

Iix Corp., Kansas City, MO

Electronic Equipment Cleaner EE-C-I7

Log \#772719

Aliphatic hydrocarbon oil.

$7 / 10 / 63$

Lockfoam Urethane Resin

Toxic - contact \& vapors (blowing agent); use isopropyl wash.

Locquic Primer Grade $T$

Main component is trichloroethylene. Also

Log \#730505 contains xylene and tetra chloroethylene.

Lotion Deodorant Skin Cleaner \#7

Contains $\mathrm{B}, \mathrm{Si}, \mathrm{Mg}, \mathrm{Na}, \mathrm{Ca} ; \mathrm{pH}=8.6$

Log \#760881

$\mathrm{CEG}$

I \& R Manufacturing Co., Kearny, NJ

I \& R \#I Watch Cleaning Solution

Petroleum distillates, N-propyl alcohol, 5-6 carbon chain amines. Boiling point $70-160^{\circ} \mathrm{C}$.

I \& R Manufacturing Co., Kearny, NJ

I \& R \#3 Watch Rinsing Solution

Petroleum distillates; boiling point $90-170^{\circ} \mathrm{C}$.

Log \#620789

$P G$

I \& $\mathrm{R}$ Manufacturing Co., Kearny, NJ

I \& $R$ \#I11 Watch Cleaner - UItrasonic

Petroleum distillates; approximately 20-25\%

ethyl alcohol, ammonia. Boiling point

$60-170^{\circ} \mathrm{C}$.

I \& R Manufacturing Co., Kearny, NJ

I \& $\mathrm{R}$ \#222 Instrument Cleaner

Petroleum distillate, n-propyl alcohol, 5-6

chain carbon amines. Boiling point $80-170^{\circ} \mathrm{C}$.

$9 / 24 / 62$

PG

$10 / 11 / 62$

PG

Luperco ACF

$50 \%$ benzoyl peroxide

Log \#620788

II 
Luperco ASF

$50 \%$ benzoyl peroxide.

Luperco ATC

$50 \%$ benzoyl peroxide with tricresyl phosphate.

Luperco CDB

$50 \%$ 2,4-dichlorobenzoyl peroxide.

Luperco $\mathrm{CSF}^{2}$

40\% 2,4-dichlorobenzoyl peroxide.

Luron Hand Soap

GSA Product No. NSN-8520-00-270-0065. pH of $1 \%$

Log \#762212 solution $=9.19 ;$ contains $0.09 \%$ lanolin. 
Macor Ceramic

Contains $\mathrm{Al}, \mathrm{Na}, \mathrm{Si}, \mathrm{B}, \mathrm{Fe}, \mathrm{Mg}, \mathrm{K}$

Maglite

Magnesium oxide.

Magna Coatings \& Chemical Corp., Los Angeles, CA

Polyurethane Coating \& Hardener

Hardener component is a polyisocyanate resin with ethyl acetate as the solvent. The coating is a polyester-vinyl copolymer wath acetone as solvent.

Magnaflux Corp.

Magnaflux

Iron particles in kerosene and toluene. Possible dermatitis hazard; respiratory hazard in high concentrations. Analysis indicated positive test for aniline content; flammable.

Magnetronics Acid Catalyst

90\% denatured alcohol (ethyl) + 10\% oxalic acid, $0.85 \mathrm{NH}^{+} ; \mathrm{pH}<1$. Use with 3240 dispersion.

Magnetronics \#3240 Thermosetting Magnetic Oxide Dispersion Iron oxide powder + amine cured epoxy resin in xylene.

Mag Oil

Mineral oil.

Malathion

0-0-dimethyl dithiophosphate of diethylmercaptosuccinate.

Mapico Red

Iron oxide coloring agent.

Mapp Gas

$22.6 \%$ propylene, $2.24 \%$ propane, $31.4 \%$ allene,

$39.0 \%$ methyl acetylene, $4.82 \%$ n-butane.

Maranite XI

No asbestos; contains natural fibers.

Marcol Oil

Flash point $347^{\circ} \mathrm{F}$ OC MC.

Marion 212 Skin Conditioners

$1.4 \%$ triethanol amine. $0.1 \%$ lanolin.
Log \#770103

CEG

Log \#711439-440

CEG

Log \#661442

LWB

$\log \# 661441$

IWB

Log \#660652

JPG

Log \#691165, 6911 691243

CEG

Log \#762309

CEG

Log \#770832 CEG 
Marlex 50

Polyethylene resin.

Marsh Adhesive C-350

Flash point $<40^{\circ} \mathrm{F}$ TCC DC.

Marsha11, John J., Co., New York, NY

Marshall's Pro-Tek-To

Log \#742350

Methyl methacrylate dissolved in toluene.

CEG

No vinyl chloride found.

Martin-Senour Primer

Zinc chromate.

Mayntane Floor Compound

Flash point $253^{\circ} \mathrm{F}, 628.20 \mathrm{~mm} \mathrm{Hg}$.

Log \#650636

MLC

Melacrone Products Co.

CX305 Cutting Fluid

Contains nitrites and amines.

CEG

Meriam \#3 Fluid

See acetylene tetrabromide.

Mertax

Mercaptobenzothiazole.

Methocel

Methyl cellulose.

Methyl Centralite

Dimethyldephenyl urea.

Methyl Tuad (Accelerator)

Tetramethylthiuram disulfide.

Methyl zymate Accelerator

Zinc dimethyldithiocarbonate.

Metriclene Solvent $\mathrm{M}-4$

25\% trichlorethylene, 53\% chlorinated

Log \#620109

hydrocarbons, remainder as high boiling

JE

carrier solvent (no aromatics).

Metriclene Solvent $\mathrm{M}-4$

A petroleum distillate of at least 6 components,

Log \#630061

aliphatic hydrocarbons plus some chlorinated

TAL solvent. Boiling point $100-160^{\circ} \mathrm{C}$ plus some high boiling residue. 
Meyercord Co., Chicago, IL

\#1505 Solution

Butyl cellosolve (Ind. Hyg. Newsletter, Vol. 5

No. 3, June, 1965).

Mica Filled Epoxy

Mica filled epoxy dust contains $\mathrm{Mn}, \mathrm{Mg}, \mathrm{Pb}, \mathrm{Fe}$,

$\mathrm{Cu}, \mathrm{Ca}, \mathrm{Na}, \mathrm{Si}$ as inorganic materials. The epoxy

Log \#710111

LWB, CEG

is an amine epoxy.

Micerotex Air Dry Coating

25\% MEK, 25\% i-propyl acetate, 25\% MIBK,

Log \#681422

$15 \%$ methylcellosolve, $10 \%$ cyclohexanone.

LWB

Micerotex Stop-Off Lacquer Reducer

$100 \% \mathrm{MEK}$.

Iog \#681423

IIWB

Micria

TIS titanium dioxide, ZR zirconium dioxide.

Micro-Cel

Synthetic calcium silicate.

Micronex

Carbon black.

Micronoil Drill and Tap Lube

Excess acid $=7.50 \mathrm{milli}$ equivalents $/ \mathrm{l}$. No

solvent distills from this product. Water

soluble, boiling point $94-96^{\circ} \mathrm{C}$ uncorrected, $\mathrm{pH} 7.4$ aqueous solution.

Micro/Type Inc., Los Angeles, CA

Big M Workover Fixative

Solvent phase - petroleum distillates methyl alcohol,

Log \#631080 TAL toluene, mixed xylenes, dichloromethane, and freon propellant. Boiling point $60-85^{\circ} \mathrm{C}$.

Miller-Stephenson Chemical Co., Inc., Danbury, CT

MS-190 HD Heavy Duty Flux Remover \& Cleaner

Log \#751757

Contains freon TMC which is an azeotrope of

CEG

freon TF and methyl chloride. Freon TF is

trichlorotrifluoro ethane. Propellent is

freon also. Ref: ACGIH Trade Names Index,

Supplement \#1 to the 1964 ed. (1966) p. 9.

Log \#620635

IL

Millipore Filter

Mn - trace, Zn - trace, Sn - trace, Bi - trace,

$\mathrm{Pb}$ - none detected. 
Military (Mil-C-37 B AM 2, 4/11/63)

Mil Spec Gun Cleaner

$0.6 \mathrm{~g}$ ammonia per liter; less than $5 \%$ aliphatic ester with the balance petroleum naphtha; no nitrobenzene.

Mineral Spirits

Flash point $107^{\circ} \mathrm{F} \mathrm{CC}$.

Min-K

No crystalline $\mathrm{SiO}_{2} \cdot$ Some fibers and glass spicules.

Min-K 1301 Insulating Material

Typical composition asbestos distributed in a fired graphite matrix. No organic binder present, will not burst into flame over bunsen burner flame or release flarmable vapors.

Min-K TE 1400

Contains calcium-magnesium silicate with small amount of $\mathrm{TiO}_{2}<10 \%$ crystalline quartz.

Min-K 2002

Supposedly silica, MgO. Analysis: $11.8 \%$ free $\mathrm{SiO}_{2}$. White, fibrous compressed mass.

Minnesota Mining \& Mfg. Co.

3M Activator

Emission spect. shows $\mathrm{Ag}, \mathrm{Mg}, \mathrm{Na}$ as cations.

Anions are nitrate, sulfate, phosphate. $\mathrm{pH}=5.25$ on original solution.

Minnesota Mining \& Mfg. Co.

3M EC-226 Adhesive

Solvent is petroleum naphtha, base is reclaimed rubber.

Minnesota Mining \& Mfg. Co.

3M Adhesive Primer

Methyl isobutyl ketone.

Minnesota Mining \& Mfg. Co.

3M Color Key Developer (Negative Acting)

By solution - H O 34\%, ethanol 66\%;

by weight - $\mathrm{H}_{2} \mathrm{O}^{2} 37 \%$, ethanol $63 \%$.

Minnesota Mining \& Mfg. Co.

3M Developer $\mathrm{R}$

Solvent is $65 \% \mathrm{H}_{2} \mathrm{O}$, 35\% ethyl n-butyl ketone.
Log \#661674

JPG

Log \#771205

LWB

Log \#641252

JPG

Log \#720864

$\mathrm{CEG}$

Log \#680981

Log \#711269

CEG

Log \#680033

JPG

Log \#761049

CEG 
Minnesota Mining \& Mfg. Co.

Log \#641293

3M Fast Bond 10 Contact Cement

90\% acetone, $6 \%$ toluene, $4 \%$ petroleum naphtha.

Minnesota Mining \& Mfg. Co.

3M Filmac, Activator

Contains acetic acid, silver, sodium sulfite. $\mathrm{pH}=5$.

Minnesota Mining \& Mfg. Co.

3M Fluorinert FC-77

Isomeric perfluorocyclic ether - probably

perfluorocyclooctylether.

Log \#660487

LWB

Log \#740503

IWB

Minnesota Mining \& Mfg. Co.

3M Scotch-Cal Thinner

This solvent is a mixture of hydrocarbons.

No halogenated products are present. A trace

of water is present; the lowest boiling

component. Decalin is present and same

aromatic components - up to $40 \%$ of the total. Remainder is a kerosene-like mixture. Boiling range $150-175^{\circ} \mathrm{C}$.

Minnesota Mining \& Mfg. Co.

$3 \mathrm{M}$ Scotchcast

Silicone rubber.

Minnesota Mining \& Mfg. Co.

3M Scotch Grip Contact Cement \#1357

Flash point $<16^{\circ} \mathrm{F}$.

Minnesota Mining \& Mfg. Co.

3M Scotch Grip Contact Cement \#2210

Solvents are acetone, toluene and hexane.

The cement is gum resin and neoprene.

Minnesota Mining \& Mfg. Co.

3M Scotchlike 700 Finisher

$35 \%$ acetone, $35 \% \mathrm{MEK}$; remainder is trace

volatiles including a freon MF propellant.

Minnesota Mining \& Mfg. Co.

Log \#640200

ROM, TAL

Log \#774191

$\mathrm{WE}$

Log \#770114

$\mathrm{CEG}$

Log \#630407

ROM

$\log \# 641253$

LWB

10\% isopropanol, $40 \% \mathrm{n}$-propanol, $50 \%$ water.

Minnesota Mining \& Mfg. Co.

Log \#730136

3M-77-Spray Adhesive

CEG

Contains as solvents a mixture of petroleum

ether and xylenes. 
Minnesota Mining \& Mfg. Co.

3M Thermofax Activator

$12 / 19 / 62$

Contains silver, acetic acid, sodium sultite

TAL

or bisulfite or thiosulfate. Probably contains

hydroquinone or paraminophenol derivatives.

$\mathrm{pH}=5$.

Mione Waterless Hand Cleaner

$12.5 \% \mathrm{H}_{2} \mathrm{O}, \sim 70 \%$ paraffin hydrocarbon,

$\sim 3 \%$ lanolin, $\sim 8 \%$ soap and detergents,

$<5 \%$ triethanol amine, $<1 \%$ perfume.

$\mathrm{pH}$ of $1 \% \operatorname{soln}=7.4$.

Miracle Black Magic Adhesive

Flash point $<41^{\circ} \mathrm{F} \mathrm{CC}, \mathrm{DC}$.

Mirid Lens Cleaner

5-10\% isopropanol and balance water; $\mathrm{pH}=7.1$.

Log \#701767

CEG

Log \#691348

CEG

ML-4 Separating Film

Methyl alcohol and methylene chloride.

MN 150 Photoresist

Contains mixed xylenes. $C \equiv N$ group present.

Log \#774187

Mobay

Mondur

Mondur $\mathrm{CB}$ : TDI + trimethylolpropane

Mondur C: $9 \%$ TDI

Mondur CA: $2 \%$ TDI

Mondur E: Ethyl isocyanate

Monour HX: Hexamethylene diisocyanate

Mondur TDS: Tolylene 2,4-diisocyanate

Mondur 0: Octadecyl isocyanate

Mondur P: Phenyl isocyanate

Mondur TM: Trimethylmethane triilocyanate

Mobay

Multrathane

Multrathane M: $p, p$ 'diphenylmethane diisocyanate.

Multrathane N5: 1,5-napthalene diisocyanate.

Mobilcer

Wax emulsion binder.

Mobile DTE-Light

Aliphatic hydrocarbon.

Log \#722798

$\mathrm{CEG}$

Mobil Mist \#27

Aliphatic hydrocarbon ester. No halogens

Log \#712562

or water present. 
Mobil Oil

Solvac $1535 \mathrm{G}$

No nitrites detected.

Log \#762314

$\mathrm{CEG}$

Mobiltherm Quenching Oil

Fire point $370^{\circ} \mathrm{F}$; flash point $347^{\circ} \mathrm{F}$.

$3 / 22 / 62$

RM

Mocoa Synthetic Oil

Contains esters and substituted benzenes.

Log \#773495

No nitrates detected.

Mogul Pb-550 Paint Stripper

Sodium phosphate and caustic soda; $\mathrm{pH}=10.75$.

Log \#650348

IWB

Molecular Sieves

Alkali metal aluminosilicates (similar to

natural clays and feld spars). (Linde

information sheet.)

Monnex \#5 44,000

Contains $\mathrm{Fe}, \mathrm{Mg}$, Si, AI as elements (talc magnesium aluminum silicate).

$12 / 19 / 72$

$\mathrm{CEG}$

Monoplex

Polystyrene resin.

Monsanto Co.

Therminol 55

Log \#772714

Material is mixed terphenyls.

$\mathrm{CEG}$

Monsanto Corp.

Therminol T-66

Log \#773927

Contains modified terphenyl.

WE

Montgomery Ward \& Co.

Log \#630063

Crown Degreaser

Contains 17.2 vol. \% isopropyl alcohol in

TAI petroleum distillate (kerosene) mixture. Not halogenated; propellant unknown. Boiling point $70^{\circ} \mathrm{C}-160^{\circ} \mathrm{C}$. Flash point $60^{\circ} \mathrm{F} \mathrm{CC}, 2 / 5 / 63 \mathrm{MC}$.

Morton's White Crystal Rock Salt

Contains $98.31 \%$ sodium chloride and $1.69 \%$ sand.

Log \#730802

CEG

Multilith Blankrola Solvent

$38.5 \%$ perchloroethylene in petroleum naphtha.

Flash point - $107^{\circ} \mathrm{F}$ CC $628.9 \mathrm{~mm} \mathrm{Hg}, 108^{\circ} \mathrm{F} \mathrm{OC}$

Log \#650713

GE, IWW $628.9 \mathrm{~mm} \mathrm{Hg}$. 
Multilith Deletion Fluid

Log \#641523 Methylene chloride.

LWB

Multilith Platex

Glycerine $+\mathrm{H}_{2} \mathrm{O}$; nickel ammonium phosphate

Iog \#641521

+ nickel ammonium nitrate.

LWB

Multilith Simflo Fountain

Glycerine + nickel ammonium phosphate + nickel

Iog \#641516

ammonium nitrate $+\mathrm{H}_{2} \mathrm{O}$.

Multilith Stop Bath

Organic acid; substituted phenolic acid.

Acid equivalent $-4.95 \mathrm{~N} ; \mathrm{pH}=0.4$.

Multilith Stop Etch

Gives test for substituted phenolic acid.

LWB

$\mathrm{pH}$ less than 2; concentrated $\mathrm{pH}$ less than 1

Log \#641261

IWB strong acid.

Murexide

Ammonium purpurate.

Mycalex

Mica-lead borate. 
Nacconal

Alkylaryl Sulfonates

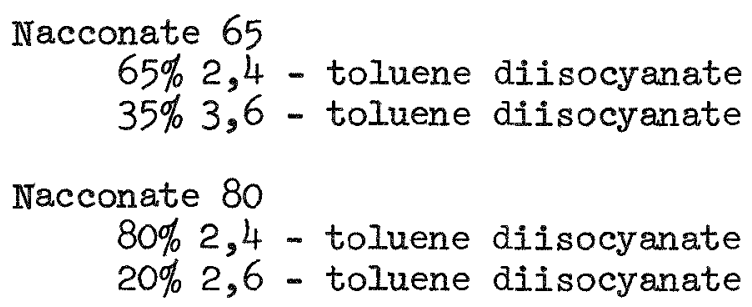

Nacconate 80

$80 \% 2,4$ - toluene diisocyanate

$20 \% 2,6$ - toluene diisocyanate

Nacconate 100

2,4 - toluene diisocyanate

Nacconate 300

Diphenylmethane - 4,4 - diisocyanate

Nacconate 310

Resin, diisocyanate

Nalclean 943

Contains trisodium phosphate, sodium carbonate,

Log \#713877

sodium bicarbonate, and soap.

Nalco Chem. Co.

Nalco 19 Sulfite

Tech. $\mathrm{Na}_{2} \mathrm{SO}_{3} ; 65 \% \mathrm{Na}_{2} \mathrm{SO}_{3}$

$17 \% \mathrm{NaCl}$

Balance $\mathrm{Na}_{2} \mathrm{SO}_{4}, \mathrm{H}_{2} \mathrm{O}$

Nalco Chem. Co.

Nalco 21 M Microbiocide

Tech. Sodium pentachlorophenolate

$\left(\mathrm{C}_{6} \mathrm{Cl}_{5} \mathrm{O}^{-} \mathrm{Na}^{+}\right)$

Log \#672864

$J P G$

Log \#672870

JPG

Iog \#661285

Nalco 21-S

Chlorinated phenolic

JPG

Nalco 25I Microbiocide

$10 \%$ solution of aliph. org. agent (amide?)

Log \#672871

in low-boiling alcohols

Nalco Chem. Co.

Nalco 39 Non Chrom

$37.7 \% \mathrm{NaNO}_{3} ; 32.4 \% \mathrm{Na}_{2} \mathrm{~B}_{4} \mathrm{O}_{7} ; 22.5 \% \mathrm{NaNO}_{2}$;

$25 \mathrm{ppm} \mathrm{Cu} ;{ }^{3}<5 \%$ surface active agent;

$<1 \%$ coloring additive

Log \#672866

JPG 
Nalco-39 Pellet

$45 \%$ inorganic as oxides, carbonates

Log \#641344

$\mathrm{Na} \mathrm{B}$ Si major constituents; AI Ti

JPG

minor constituents, $\mathrm{V}, \mathrm{Cu}$, Mg trace constituents.

Elements not detected <.I\% $\mathrm{Pb}, \mathrm{Fe}, \mathrm{Cr}$, $\mathrm{Co}$,

$\mathrm{Mn}, \mathrm{Ni}, \mathrm{Sb}, \mathrm{Bi}, \mathrm{Ba}, \mathrm{K}, \mathrm{Ag}, \mathrm{Zr}$

Nalco 205

Kerosene base brominated hydrocarbon

Log \#661284

JPG

Nalco Chem. Co.

Nalco 205 Microbiocide

Unidentified, probably a Di-N subst. arom.

Log \#672867

JPG amine

Nalco \#205

Product is brominated kerosene

Log \#715590

CEG

Nalco 207

Mfgr - 10\% methylene Bisthiocyanate 90\% Inert

IR verification

Nalco Chem. Co.

Nalco 237 Bactericide

Log \#672872

Tech. $\mathrm{Na}_{2} \mathrm{CrO}_{4}$

Assay: $11.46 \% \mathrm{Na}$

$20.61 \% \mathrm{Cr}$

$65 \% \mathrm{Na}_{2} \mathrm{CrO}_{4}$

Nalco 295

Aliphatic sulfonic acid

Log \#661287

JPG

JPG

Nalco Chem. Co.

Nalco 295 PH Control

Tech. citric acid

Log \#661286

JPG

Nalco Chemical Co.

Nalco 356

Contains cyclohexylamine and morphaline.

Log \#672872

JPG

Supplier Informati

Nalco Co.

Nalco 356

Log \#772727

Material is $65 \%$ ethylene diamine/35\% unknown

CEG

primary amine. 
Nalco Chem. Co.

Log \#672865

Nalco 356 Amine

JPG

Mixture of two short-chain primary

amines; $65 \%$ ethylene diamine $\left(\mathrm{H}_{2} \mathrm{~N}-\mathrm{CH}_{2} \mathrm{CH}_{2}-\mathrm{NH}_{2}\right)$

$35 \%$ unknown pri. amine

Nalco Chem.

Nalco 390

$4.5 \% \mathrm{Na}, 4.5 \% \mathrm{Zn}$, polyglycol ether surface active agent

Iog \#672834

JPG

Nalco 390 Phosphate

Contains ions $\mathrm{Zn}^{++}, \mathrm{Na}^{+}$, sulfate, thiosulfate

Log \#692377 and phosphate

Nalco Chem. Co.

Nalco 718 Phosphate

Tech. $\mathrm{Na}_{2} \mathrm{H} \mathrm{PO}_{4}$

CEG/EG

$26.6 \% \mathrm{Na}$

$17.8 \% \mathrm{P}$

$610 \mathrm{ppm} \mathrm{Fe}$

$70 \% \mathrm{Na}_{2} \mathrm{HPO}_{4}$

Nalco Chem. Co.

Nalco 751 Organic Antifoam

Tech. sodium alkyl benzene sulfonate

Log \#672863

JPG

Nalco Chem. Co. - Chicago, IL

Nalco \#753

$42.5 \% \mathrm{Na}_{3} \mathrm{PO}_{4}$

5\% oxyethylated alcohol (biodegradable

surface active agent); $52.5 \% \mathrm{NaOH}$

Nalco Chem. Co.

Nalco \#755

$62 \% \mathrm{NaOH} ; 38 \%$ oxyethylated alcohol (biodegradable surface active agent)

Nalco Chem. Co.

Nalco 820 Polyphos

Log \#672869

$9 \% \mathrm{Na}_{2} \mathrm{HPO}_{4}$; remainder is $\mathrm{Na}$ salt of a

Log \#672868

JPG

Log \#680879

Log \#680880

surface-active agent (organic) 
Nalco Co., Chicago, II

Nalco 7325 Water Treatment

Log \#740719

Material is an organo-tin compound in an

$\mathrm{CEG}$

aliphatic hydrocarbon solvent.

Nalco 7350

Material is a water-soluble primary amine.

Log \#772715

$\mathrm{CEG}$

Nalco Chloro-phenate Boiler Compound

Also a polyphosphate.

Napco Chemical

Lockfoam A-2IO-R Accelerator ( $T$ Component)

Tolylene diisocyanate. Gives of carbon

dioxide and cyanicacid when mixed with resin.

Contact with moisture causes the formation

of amines and polyurethanes. $R$ component is

a polyester, relatively harmless. Use isopropyl

alcohol to clean up spills.

Naptha Cleaner

$40 \%$ methyl chloroform $+60 \%$ naphtha cleaner $95^{\circ} \mathrm{F}$

$20 \%$ methyl chloroform + $80 \%$ naphtha cleaner $119^{\circ} \mathrm{F}$

$10 \%$ methyl chloroform $+90 \%$ naphtha cleaner $109^{\circ} \mathrm{F}$

Iog \#650913

GE

National Aniline Corp.

Methyl Malic Anhydride

Malic acid adduct of phthalic anhydride.

National Chemsearch Corp.

SS-25 Ready-To-Use Solvent Degreaser

$12 \%$ dichloromethane, $88 \%$ petroleum naphtha.

$\mathbb{N}_{D} 1.4405$; boiling point $90-115^{\circ} \mathrm{C}$; flash point for petroleum naphtha $40^{\circ} \mathrm{C}$.

National Chemsearch Corp.

$\log \# 650411$

SS-25 Solvent Degreaser

10\% methylene chloride, $90 \%$ petroleum naphtha.

$10 / 3 / 61$

JE

National Lead

Barinal

Barium Ricinoleate - a primary plastisol

system stabilizer.

National Lead Co.

Bentone 34

Organophillic Bentanite - a flow control agent for plastisols. 
National Lead Co.

Dyphos

Lead Monohydrogen phosphite weathering

stabilizer for vinyl chlorides.

National Lead Co.

Nalcosol TH 53 \& TH 48

$53 \%$ toluene in petroleum distillate hydrocarbon

$11 / 29 / 61$

JE mixture. TH $535 \%$ toluene, rest similar to $\mathrm{TH} 48$. Boiling point $50-70^{\circ} \mathrm{C}$; toluene flash point $6^{\circ} \mathrm{C}$.

Nashua 2400 Toner

Acrylic resin with carbon black.

Log \#690262

EG

Naugatuck Chemical Co.

Vibrin

Polyester styrene.

Neogard Corp. (Jones Blair)

Neogard 7013 White Hypalon

Log \#752516

Solid material is neoprene elastomer. Solvent

CEG

is xylene.

Neozone A

Phenyl-alpha-naphthylamine.

Neozone D

Phenyl-beta-naphthylamine.

Neutra-Clean \#7

$\mathrm{pH}$ when cold $=7-8, \mathrm{pH}$ at $160^{\circ} \mathrm{F}=7-8$,

Log \#641345

$\mathrm{pH}$ at $205^{\circ} \mathrm{F}=7-8$; no fumes or noxious

JPG

smell ( $120 \mathrm{oz} / \mathrm{gal} \mathrm{H}_{2} \mathrm{O}$ ).

Neutra-Clean E-7

$10 \%$ sodium thiosulfate, less than $1 \%$ sodium

Log \#661757

sulfate, a trace of wetting agent and the

JPG

balance sulfamic acid. Gives off sulfur

dioxide when made acid at $150^{\circ} \mathrm{F}$.

Neu-Tri

Trichloroethylene.

New England Tape Co.

Adhesive 11-9(SCS-11)

Vinyl plastic in solution composed of $60 \%$ cyclohexanone and $40 \%$ tetrahydrofurane. 
New Mexico 1st Quality Paint Co.

Interior-Exterior Semi-Gloss Enamel, White

Log \#771408

Contains aliphatic hydrocarbon solvent.

$\mathrm{CEG}$

Boiling point $190^{\circ}-240^{\circ} \mathrm{C}$. No aromatic or chlorinated solvents.

Ney-Oro- "G" Wire

Gold, platinum, silver and copper.

Nicusil

$<0.05 \% \mathrm{Cd}$.

Iog \#661526

Nipx

Poly glycols (thickens); catalyst - dibutyltin dialaurate; propylene glycol.

North American Mogul Products Co.

MoguI PB-592

pH of $1 \%$ solution $=12.8$. Emission shows

$\mathrm{Na}, \mathrm{Ca}, \mathrm{Si}$ as major elements. No phosphates. Possibly tech. grade $\mathrm{NaOH}$, also has insoluble silicates. Also contains calcium probably as hydroxide.

Nu-Coil Compound

A scale-removing cleaning compound.

$\mathrm{Nu}-\mathrm{COII} 40$

Dinonyl phthalate.

Nyclad \#584 Stripper

A U.S.P.X. mixture of isomeric cresols; otherwise

Log \#630875 named "creotex" or creosote oil. Poison and

TAL

Log \#750255 $\mathrm{CEG}$

vapor hazard - $5 \mathrm{ppm} \mathrm{MAC.}$

Nylon

Polyamide. 
Octagon Process, Inc., Edgewater, NJ

Cleaning Compound.

Log \#650809

Chlorinated petroleum distillate.

Flash point $185^{\circ} \mathrm{F}$ at $626.9 \mathrm{~mm} \mathrm{Hg}$.

Octagon Process Inc., Edgewater, NJ

Duplicating Fluid \#9800

95\% $\mathrm{CH}_{3} \mathrm{OH}, 5 \%$ carbitol. "7510-272-9800

Log \#670254

Direct Process Spirit Type O-D-825B AMI

Type II GS-005-40617." $\mathrm{N}_{D}=1.3439$ (18);

flash point $50^{\circ} \mathrm{F}$ OC; flammability $20-70^{\circ} \mathrm{F}$.

Octagon Process, Inc., Edgewater, NJ

Duplicating Fluid \#9802

95\% ethyl alcohol, $5 \% \mathrm{CH}_{3} \mathrm{OH}$. "Contains methyl alcohol." "7510-272-9802 Direct

IWB

Process Spirit Type OD-825B AMI Type I

GS-005-40617." Flash point $58^{\circ} \mathrm{F} \mathrm{OC;}$

flammability $20-70^{\circ} \mathrm{F} ; \mathrm{N}_{\mathrm{D}}=1.3637(18)$.

Ode1l, M. P., Co., Cleveland, $\mathrm{OH}$

San-Del \#2 Tech. Cleaner Cone

Material is a syntehtic detergent. Contains

$\mathrm{P}, \mathrm{Na}, \mathrm{Si}$, probably as various compounds such

as trisodium phosphate, sodium silicate, etc.

$\mathrm{pH}$ of cone. soln. $=9$.

OId Town Corp., Brooklyn, NY

Carbon Paper Cobalt Blue Process

Mostly organic dyes, wax binders and carbon

black. No organic volatile solvents or

inorganic toxic materials were found.

Old Town Corp., Brooklyn, Nr

Cobalt Blue Process Fluid

95\% $\mathrm{CH}_{3} \mathrm{OH}, 5 \%$ carbitol [TNI 95\% $\mathrm{CH}_{3} \mathrm{OH}+$

(glycol ether $+\mathrm{H}_{2} \mathrm{O}$ ) $5 \%$ ]. $\mathrm{N}_{\mathrm{D}}=1.3476(18)$.

"Contains methanol." Flash point $54^{\circ} \mathrm{F}$ OC;

flammability $20-70^{\circ} \mathrm{F}$.

Log \#670255

LWB

Log \#742968

CEG

Log \#641137

JPG

Ortho

Leaf Polish Bomb ( $1 / 2$ pt. aerosel can)

Three component mixture containing 90-95\%

isopropyl alcohol; two low boiling traces

plus a fluorocarbon propellant.

Ozalid Mylar Cleaner

pH 7.2

Iog \#630600

TAI 
Packwood, G. H., Mfg. Co.

Pax Calgon \#3.

$2.75 \%$ lanolin, minor borax, minor silicates, fair spectral purity, pH of $1 \%$ slurry - 9.6; $\%$ insoluble in $1 \%$ slurry - 50\%. Odor - soapy, strong; particle size uniformity - fair; dust generation when poured - some.

Paint Thinner

Usually a hydrocarbon such as turpentine, hydrogenated naphthalene or some relatively nonvolatile petroleum fraction; flammable vapor hazard.

Paint Thinner Reducer \#8

$100 \%$ toluene; flash point $52^{\circ} \mathrm{F}$ OC.

Paint Thinner - T831 Dual Purpose

$35 \%$ acetone, $5 \%$ methylethyl ketone, $10 \%$

n-butyl alcohol, $15 \%$ toluene, $20 \%$ petroleum

naphtha and $15 \%$ cellosolve acetate. Flash point less than $18^{\circ} \mathrm{F}$ OC; flammability $<20^{\circ} \mathrm{F}$.

Pale Kavri Resin

A lacquer - forms a hard film.

Paliney Metal

A mixture of noble metals, including copper, nickel, rhodium, palladium, platinum, gold and silver.

Palmer Co.

UCC Chemical \& Plastic

Material is a silicone oil.

PAPI

Isocyanate mixture, hardener for polyurethane foam resins.

Paraffin Wax

Flammable material and moderate fire hazard.

Parker Unipor Compound

A thread and gasket compound.

Peck's Product Co.

Pepco \#415 Hand Soap

No lanolin or lecithin detected. Emission shows $\mathrm{B}, \mathrm{K}, \mathrm{Na}, \mathrm{Al}, \mathrm{Si}$. Probably in the form of soap, pumice and borax. $\mathrm{pH}$ of $1 \%$ solution $=7.9$.
Log \#672514

JPG

Log \#661504

JPG

Log \#661503

JPG

Log \#772712

CEG

Log \#721956

CEG 
$P$ \& E Cleaner

$3.6 \% \mathrm{HCl}, 7.2 \%$ oxalic acid, trace detergent,

$\log \# 680053$

trace deodorant, balance water.

JPG

Pelargonic Morpholide

Relatively non-toxic, but highly irritating to

the eyes and respiratory tract. Estimated

maximum safe inhalation dose $=50 \mathrm{mg}$-min.

No ill after effects.

$m^{3}$

Penn Scientific Product Co., Abington, PA

Spectrum Diamond Lapping OiI

Log \#762659

Material is an ester based aliphatic

CEG

hydrocarbon oil.

Pershing Tube Mock HE

$\mathrm{Si}, \mathrm{Cu}, \mathrm{P}$ only elements detected.

Log \#770578

CEG

Petrosul

Grease.

Phillips Petroleum Co.

Log \#772726

All Seasons Motor Oil

CEG

Material is an aliphatic hydrocarbon oil.

Photo-Foil Etching Powder

Potassium hydroxide.

\#747 Photoresist

Contains mixed xylenes. $\mathrm{C}=\mathrm{N}$ group present.

Log \#774189

WE

\#747 Photoresist Developer

Contains mixed xylenes and aliphatic

Log \#774184

hydrocarbons.

\#1350 Photoresist Developer

Nitrites present, mainly $\mathrm{H}_{2} \mathrm{O}$.

Log \#774185

WE

\#1350 J Photoresist

Functional groups: ester, $\mathrm{C} \equiv \mathrm{N}$, high $\mathrm{H}_{2} \mathrm{O}$

Log \#774186 content.

$\mathrm{WE}$

Pilot B Plastic

Polyvinyl toluene.

Log \#661686

JPG

Pink Glow Cleaner for Bowl \& Porcelain

$51.2 \% \mathrm{H}_{2} \mathrm{O}, 20.8 \% \mathrm{PO}_{4} \equiv$ as $\mathrm{H}_{3} \mathrm{PO}_{4}, \sim 7 \%$ soaps and detergent, $\sim 21 \%$ high Boiling $\left(>200^{\circ} \mathrm{C}\right)$

Log \#715109

CEG

hydrocarbon mixture. $\mathrm{pH}$ of original $=1.25$. 
Pittsburgh Paint Co.

Interior Alkyd Enamel LoSheen \#54-21

Log \#761171

Mixture of aliphatic hydrocarbons and xylene.

CEG

Pittsburgh Paint Co.

Paint \& Varnish Remover

$58 \% \mathrm{CH}_{2} \mathrm{Cl}_{2}$ by volume + high boiling viscous

Log \#640335

ROM liquid. Flash point $84^{\circ} \pm 10^{\circ} \mathrm{F}$ CC ( $\log \# 640340$, ROM).

Pittsburgh Paint Co.

Paint \& Varnish Remover (75-10)

$77.8 \%$ methylene chloride, $18.1 \%$ methanol,

$4.02 \%$ dichloroethane in a paraffin wax base.

Pittsburgh Paint Co.

Selectron

Unsaturated polyester resins.

Pittsburgh Paint Co.

Wallhide Semi-Gloss Enamel Interior 27-109 White Contains aliphatic hydrocarbon solvent; boiling point $130^{\circ}-210^{\circ} \mathrm{C}$; no aromatic or chlorinated solvents.

Plastic Bonded Explosives

See nitrocellulose, chloroethylphosphate, trimethylenitrinitroamine, and tetramethylenetetranitroamine.

Plast-I-Kleen (Plastic Cleaner)

Nontoxic, a liquid detergent.

Log \#641339

LWB

Plast-X-Cote Paint

Part A - volatiles unknown, contains pine oil;

$\log \# 692603$

$\mathrm{CEG}$

Log \#771407

CEG major components - a vinyl epoxy compound; vinyl

$11 / 13 / 63$

TAL epoxy material.

Plibond \#30 Cement

$30 \%$ solids dissolved in methyl ethyl ketone.

Pliolite

Styrene-buladiene rubber.

Pluracol TP

Polyglycol - flexiblizer.

Polyophen

Phenolic resins. 
Polyox Resin

A water soluble resin; contains ethylene oxide.

Poly-Prep

Primer, polyethylene treating; contains

$\mathrm{H}_{2} \mathrm{SO}_{4} \cdot$

Polystyrene 0 , Dope Thinner

Flash point $<42^{\circ} \mathrm{F} \mathrm{CC}$.

Prepolymer Mix

Toluene diisocyanate and castor oil.

Price Research Ltd., Kansas City, MO

Uniclean

$90 \% 1,1,1-t r i c h l o r o e t h a n e, 10 \%$ methyl

Log \#661805

phenyl polysiloxane. TLV 350 ppm;

flammability $>200^{\circ} \mathrm{F}$.

Price Research Ltd., Kansas City, MO

Uni-pol Light Duty Linoleum Cleaner

Contains $35.6 \%$ water and $64 \%$ kerosene with

Log \#770083

a trace of wax and $0.3 \%$ soap. GSA Item No.

$C E G$

7930-579-8631. Flash point $190^{\circ} \mathrm{F}$ or $87^{\circ} \mathrm{C}$

OC.

Products Research Co.

PRC

Epoxy sealants.

Products Research Co.

PRC Hardener

Synthetic rubber sealing compound.

Pro-Seal

Polysulfide sealer.

PSI-10 Aerosol

Hydrocarbon oil plus a freon gas propellant.

$\log \# 691494$

LWB

Pyrene G-I

Powdered graphite.

PZT Ceramic

Lead oxide, titanium oxide, zirconium oxide, and niobium oxide. Made in Building 840. 
Quickmount Liquid

Epoxide structure with non-conjugated

Log \#630349

double bond, probable carbonyl group.

TAI

$\mathrm{Sp} . \mathrm{Gr} .=0.9775 @ 22^{\circ} \mathrm{C}$. Ind. Ref. =

1.4165. Boiling point $93^{\circ} \mathrm{C}$.

Quietrol Cleaner \& Lubricant

$95+\% 1,1,1-$ trichloroethane $\pm 5 \%$ light oil

$\log \# 630388$

and coloring agent.

Quietrole Cleaner \& Lubricant

95\% 1,1,1-trichloroethane, 5\% light mineral

oil + red color agent.

TAI,

Quixae

5 gallon methyl alcohol, 100 gallon ethyl alcohol.

Log \#680878

LWB 
Radio Service Solvent

$5 \%$ ethanol, 35\% isopropanol, 35\% ethyl

Log \#660709

acetate, $20 \%$ isobutyl acetate.

Ram Chemicals Inc., Gardena, CA

Desolv 292

Dichloromethane (methylene chloride) and

an organic dye base; $500 \mathrm{ppm}$. Used to soften

epoxy for stripping. Ref: N. J. DeLollis, 1112-2.

Raychem Flamtrol

12 AWG TCW-600V 90C 1976. Copper wire $\mathrm{Pb}, \mathrm{Fe}$,

Log \#772597

$\mathrm{Sb}, \mathrm{Si}$ in wire coating.

Ray K-12, Ray K-16 Dielectric Blocks

Major constituent TiO; minor constituent Si, P;

traces of $\mathrm{Al}, \mathrm{Fe}, \mathrm{Mg},{ }^{\mathrm{V}} \mathrm{V}, \mathrm{Sn}, \mathrm{B}, \mathrm{Cd}, \mathrm{Ca}, \mathrm{Cu}$.

$\log \# 641262$

R Developer

Contains $85 \%$ solvent ( $70 \% \mathrm{H}_{2} \mathrm{O}$ and $30 \%$-heptanone), $15 \%$ residue.

Recordak Dacomatic 526 Bleach Product \#4360

Contains sulfuric acid, potassium fericyanide

and potassium dichromate.

Recordak Dacomatic 526 Clearing Bath Product \#4348

Contains ammonium hydroxide, sodium or potassium

hydroxide. Mostly water; $\mathrm{pH}=10.0$.

Recordak Dacomatic 526 First Developer Product \#4346 Contains sodium sulfite, hydroquinone, sodium hydroxide, potassium bromide, sodium carbonate; $\mathrm{pH}=13.4$.

Recordak Dacomatic 526 Redeveloper Product \#4349 Contains sodium sulfite, sodium hydroxide, $\mathrm{KBr}$, $\mathrm{Na}_{2} \mathrm{CO}_{3}$ and hydroquinone; $\mathrm{pH}=13.6$.

$4 / 7 / 64$

108 \#1262

Log \#740585

LWB

Log \#713376

CEG, EL

Log \#713375

CEG

Log \#713373

Recordak Monobath 40

Sodium thiocyanate, sodium sulfite, paraphenylenediamine (caustic, skin irritant) or parahydroxyphenyl glycine (or derivatives). May contain potassium bromide. Aqueous $\mathrm{pH}=9$.

Log \#713374 CEG

$12 / 19 / 62$

TAL

Reducer (for T831 Thinner)

$100 \%$ toluene; flash point $52^{\circ} \mathrm{F}$ OC.

$\log \# 661504$

Relton Corp., Arcadia, CA

Log \#692939

Rapid Tap Cutting Fluid

LWB

$92 \% 1,1,1$-trichloroethane, $7.5 \%$ hydrocarbon oil, $0.5 \%$ aliphatic ester. 
Resistor Paste PPL-4

Contains $8.26 \%$ Tl. Resin insol. in DMF, acetone, $\mathrm{CCl}_{4}$, benzene, ether, ethanol. Resin insol. in $\mathrm{H}_{2} \mathrm{O}, 2 \mathrm{NHNO}_{3}$. Brown amorphous mass.

Revere Ice-Melter

Major constituents $\mathrm{AlCl}_{3}, \mathrm{CaCl}_{2}, \mathrm{NaCl}$, trace

$\mathrm{BaCl}_{2}$. All components anhydrous, producing heat when allowed to come into contact with $\mathrm{H}_{2} \mathrm{O}$ or ice. White rounded pellets.

Rex Corp.

Rexilite 1422

Plastic, contains styrene monomer. When turning the hardened product, a coolant is recommended.

Rezolin R-72 S

An acid cured phenolic resin. Acid may be either toluene sulfonic or benzene sulfonic. Chips are corrosive to tools.

$\mathrm{RF}-300$ Resin Formulation Contains pet. ether. Major component is n-hexane (52.3\%). Minor components (4) are a mixture of hexane or pentane isomers.

Rigidex

High density polyethylene.

Log \#641415 JPG

Log \#700038

CEG

Rohm \& Haas

Paraplex

Polyester resins.

Rogers Molding Compound

A diallyl phthalate resin containing glass fibers of varying lengths. Hardener is a peroxide which is incorporated in the resin and cures under heat. No mixing is involved.

Roller Cleaner \#360

$20 \%$ methyl chloroform $+80 \%$ petroleum naphtha

(aliphatic); flash point $92^{\circ} \mathrm{F}$.

Rollerwash

Contains aliphatic hydrocarbons and tetra

chloroethylene.

Rotanium Belt Grip

Contains mineral oil, 1,1,1-trichloroethane and

Log \#650518

a benzoate as preservative. The propellant is

Log \#693019

LWB

1,1,1-trichloroethane and the "liquid" is mineral oil. 
Rotax

Mercaptobenzothiazole.

RTV Silicone Rubber

Log \#651100

Silicone rubber and acetic acid.

LWB

Rustlick Inc.

Log \#762315

Rustlick G25AH

CEG

Contains nitrites.

Log \#770102

Rustiick Inc.

Rustlick G25AH

Teritiary amines with unknown diazonium salts

$(\mathrm{R}-\mathrm{N} \equiv \mathrm{N})$. $\mathrm{pH}$ of undiluted material is 9.5.

Emission spec. shows the elements $\mathrm{Na}$, $\mathrm{Si}, \mathrm{Fe}, \mathrm{Mg}$. 
Saco Electrical Cleaner \#37

$2 \%$ methylene chloride, $7 \%$ 1,1,1-trichloroethane,

$\log \# 641121$

$1 \%$ tetra chloroethylene, $90 \%$ petroleum distillate

LWB

of the kerosene range.

SAE \#10 Lub. Oil

Flash point $445^{\circ} \mathrm{F}$ (DC).

SAE \#2O Lub. Oil

Flash point $460^{\circ} \mathrm{F}$ OC (DC).

SAE \#30 Lub. Oil

Flash point $470^{\circ} \mathrm{F}$ oC (DC).

SAF Acid

The anion is sulfate $\left(\mathrm{SO}_{4}^{-}\right) ; \mathrm{pH}=1.2$. One

gm of acid required $95 \mathrm{mI}$ of $0.1 \mathrm{~N} \mathrm{NaOH}$ for

neutralization.

Sandeze Solvent

A solvent used in Area V. Impure mixture

of ethanol $\left(\mathrm{C}_{2} \mathrm{H}_{5} \mathrm{OH}\right)$ and methyl acetate, plus

3 impurity fractions of higher boiling point.

Log \#650044

LWB

Savogram Co., Norwood, MA

Kutzit

Paint stripper; acetone, methyl alcohol and

more than 15\% benzene; flammable and toxic.

SBS-30 Waterless Hand Cleaner

$8.3 \% \mathrm{H}_{2} \mathrm{O}, \sim 75 \%$ paraffin hydrocarbons, $\sim 3 \%$

lanolin, $\sim 8 \%$ soap and detergent, $<5 \%$

triethanolamine, $<1 \%$ perfume. $\mathrm{pH}$ of $1 \%$

$\operatorname{soln}=7.15$.

SBS-30 Waterless Skin Cleaner

Composed of glycine, fatty acid esters, water

Log \#640173

ROM, TAI and lanolin. $\mathrm{pH}$ of $1 \%$ soln $=7.15$. (Breakdown same as above).

SBS-40 Medicated Skin Cream

Composed of a silicone oil and fatty acid ester.

Log \#691619 Also contains water and lanolin.

Log \#701766 $\mathrm{CEG}$

Log \#691621

CEG

CEG

SBS-40 Medicated Skin Cream

$2.6 \%$ triethanol amine, $0.09 \%$ lanolin.

Log \#770833

$\mathrm{CEG}$

SBS-4I Medicated Skin Lotion

Composed of a fatty acid ester, lanolin,

Log \#691620 water and glycine. 
SBS Vegetable Base Hand Cleaner \#1l

Contains $\mathrm{Si}, \mathrm{Mg}, \mathrm{Na}, \mathrm{Ca}, \mathrm{Fe}, \mathrm{K} ; \mathrm{pH}=8$.

Log \#760879

CEG

Schnee-Morehead Chemicals, Inc., Irving, TX

Acryl-R \#5505 Seam Sealer

Acrylic ester plus xylene $(<3 \%)$.

Log \#771256

$\mathrm{CEG}$

Se Rectifiers

641397-99 Washers (3) New - H.D. (<0.1 mg Se)

641400-02 Plates (3) New - Avg. $349.6 \mathrm{mg}$ Se

641403-05 Units (3) New - Avg. $364.5 \mathrm{mg} \mathrm{Se}$

641406-08 Washers (3) Burned Avg. $10 \mathrm{mg}$ Se

641409-11 Plates (3) Burned Avg. 317.6 mg Se

641412-14 Units (3) Burned $320.6 \mathrm{mg}$ Se

Shaklee Algicide Concentrate

Triethanolamine hydrochloride; $\mathrm{pH}$ (water solution) = 6.0

Shaklee Basic I Cleaner

Unreacted alkali (diluted solution) - $0.25 \mathrm{ml}$

of $1 \mathrm{~N} \mathrm{H}_{2} \mathrm{SO}_{4}$. $\mathrm{pH}$ concentrate $=11.4 ; \mathrm{pH}$ diluted

$1 \mathrm{oz} / \mathrm{gal}^{2}=10.1$.

Log \#670430

JPG

Log \#641346

LWB

Sharp Black Carbon Paper

Mostly organic dyes, wax binders and carbon

black. No organic volatile solvents or inorganic

toxic materials were found.

Shell Oil Co.

DIALA "AX" Transformer Oil

Contains aliphatic hydrocarbon oil. No elements

detected, no silicone detected.

Shell Oil Co.

Epon Curing Agent "Z"

Material is epoxy resin of diphenyl propyl

amine. Hardener is a curing agent composed.

of p-phenylene diamine and 2,3,5-tri(dimethylamine)

methyl phenol.

Log \#641139

JPG

Log \#772246

CEG

Log \#752049

$\mathrm{CEG}$

Sherwin Williams

Acrylic Lacquer Thinner $57 \mathrm{~K} 248$

Contains $24.4 \%$ toluene; $56.3 \%$ ethyl acetate;

Log \#740376 $19.3 \%$ alcohol.

Sherwin Williams

Dypol

Polyester resin. 
Sherwin Williams

Paint Thinner

Contains Xylene.

Sherwin Williams

"Sher-Cool" TMFC-90

CEG

Contains nitrites and amines.

$\log \# 651320$

$11 \%$ propyl alcohol, $36 \%$ propyl acetate, 53\%

polyacrylonitrite and amide plastics.

Shipley AZ Resist 1350

$+3.8 \%$ n-propyl acetate, $0.9 \%$ isopropanol,

$5 / 16 / 68$

$37.6 \%$ cellosolve acetate, $4.7 \%$ n-propanol, $53 \%$ polymethyl acrylate plastic.

Shipley AZ $1350 \mathrm{H}$ Azoplate

Inorganic - Na. Solvent - ethylene glycol

monomethyl ether acetate. Plastic - polyacrylamide and isoprene copolymer.

Shipley IT 26 Tin

Contains $\mathrm{Sn}, \mathrm{Mg}, \mathrm{Na}, \mathrm{P} . \mathrm{Sn}, \mathrm{P}$ - major;

$\log \# 751969$

$\mathrm{Mg}, \mathrm{Na}$ - minor.

Silastic 140 Adhesive

Silicone rubber + acetic acid as possible

Log \#702388

CEG curing agent.

Sil-Bond

Silver Brazing Alloy

\#45(0.062) $22.6 \% \mathrm{Ca}$.

Log \#660531

Silver Solders

Silverloy $355-0.1 \% \mathrm{Cd}$

Silverloy $50-17.8 \% \mathrm{Cd}$

Easy Flow Wire $1 / 32-30.1 \% \mathrm{Cd}$

Easy Flow Sheet - $21.0 \% \mathrm{Cd}$

Sil-Bond 45 Wire $(.062)-22.6 \% \mathrm{~cd}$

Aircosil $45-23.3 \% \mathrm{Ca}$

Allstate 105 - $98 \% \mathrm{Cd}$

Incusil 10 - less than $0.05 \% \mathrm{Cd}$

Nicusil 3-less than $0.05 \% \mathrm{Cd}$

Allstate 430 - less than $0.05 \% \mathrm{Cd}$

Silver Q Flux

$0.0043 \% \mathrm{~F}^{-}$

Log \#660679

JPG 
Sinclair "Strip-Clean" 171

$70.8 \%$ methylene chloride, $25.7 \%$ methanol,

Log \#692583

$4.5 \%$ 1,2-dichloroethene; in a paraffin wax base.

Smith Corona Copier 111 Toner

Emission shows carbon only. Infrared shows only a mixture of aromatic and aliphatic hydrocarbons. Black material in suspension is carbon black.

Smith Corona Marchant

Microstatic Replenisher and Copy Fluid

Copy Fluid - aliphatic organic mixture containing some primary or secondary alcohol; no aromatic, no halogen and no water ( 6 or 7 components). Microstatic Replenisher - Same as above, but with aromatic coloring agent of low composition temp.; indicates postest for pri-sec alcohol, neg. halogen and neg. $\mathrm{H}_{2} \mathrm{O}$. Color agent $10 \%(\mathrm{v} / \mathrm{v})$ of total. Boiling range $136.4-147.5^{\circ} \mathrm{C}$.

Smith Corona Marchant

Stat Dispersant-B

High boiling aliphatics (mineral spirits).

Flash point $117^{\circ} \mathrm{F}$ OC.

Smith Corona Marchant

Toner Mix

Material is an aliphatic hydrocarbon mixture

with carbon black.

Snopake Fluid

Volatile fraction contains 5 low boiling components in trace amounts, plus toluene. The residue is hard, dry white pigment - like dried paint.

Snopake Thinner \& Brush Cleaner

Toluene is solvent.

$\log \# 621044$

Soap Powder (Orange colored)

$\mathrm{pH}=10.8$; acid equivalent $=6.65 \mathrm{ml}$ of $I \mathrm{~N}$ $\mathrm{H}_{2} \mathrm{SO}_{4} / \mathrm{gm}$ soap.

Log \#631049

TAI

$11 / 19 / 62$

TAL

Log \#680355

Log \#741122

CEG

Log \#650043

LWB

Solox

Shellac and paint solvent consisting of ethyl and methyl alcohols.

Soluvac Water Soluble Oil

Hydroxy aliphatic hydrocarbon ester. No

Log \#712561

halogens present.

$\mathrm{CEG}$ 
Solvasol

Solvasols 1-6 are naphtha (petroleum)

first grade. The second grade, Solvasols

73-75, are high power solvent naphthas.

Flash point $121^{\circ} \mathrm{F} \mathrm{CC}$.

Solventol - Powdered Toilet Soap

Meg. alkali $=28.76 \mathrm{mI} \mathrm{I} \mathrm{N} \mathrm{H} 2 \mathrm{SO}_{4} / \mathrm{gm}$ of soap.

Log \#641260

$\mathrm{pH} 8.8$.

LWB

Sour

Ammonium silicofluoride. Used in radioactive decontamination rinses.

Sparkle Window Cleaner Ammonium bifluoride (acid amm. fluoride); see amm. fluoride.

Speed-O-Print Business Machine Corp., Chicago, III. Spirit Duplicating Fluid $\mathrm{CH}_{3} \mathrm{OH}$. SBM \#2500 "Fluid Pak . . for all liquid duplicating machine." "Contains wood alcohol (methanol)." $\mathrm{N}_{\mathrm{D}}=1.3290$ (18); flash point $52^{\circ} \mathrm{F}$ OC; TLV $200 \mathrm{ppm} ; \mathrm{mg} / \mathrm{m} 3 ;$ flammability $20^{\circ}-70^{\circ} \mathrm{F}$.

Spot Remover

$49 \%$ (vol.) toluene, 47\% (vol.) carbon tetrachloride, 3\% (vol.) unknown trace constituent (high boiling).

Spra Kleen (Electrical Contact Cleaner \& Lubricant) 95\% methyl chloroform, 5\% methylene chloride, trace oil, trace odorant, propellant Freon 12.

Log \#730859 LWB

Spray Mist

Aqueous solution - $\mathrm{H}_{\mathrm{O}} \mathrm{O}$ distills leaving colored residue. $\mathrm{pH}=10.0$ solution, 9.3 distillate,

Log \#670249 LWB 10.0 residue.

Sta-Coat

$8 \%$ hexachlorophene, $2 \%$ detergent, trace deodorant

Log \#661645 and the balance water.

Log \#630031 TAL

Staeh, Wm. M., Co. Inc.

$\mathrm{Hg} \mathrm{X}$ Mercury Decontamination 50-60\% sodium thiosulfate, balance is sodium dithionate $\left(\mathrm{Na}_{2} \mathrm{~S}_{2} \mathrm{O}_{6}\right)$. 
Standard 100-Petroleum Distillate Boiling point range $53-175^{\circ} \mathrm{C}$.

Log \#722799

$\mathrm{CEG}$

Staramic

Starch base binder.

Starch

Has been known to cause allergic symptoms

in humans.

Stat-King Processing Solution - Black Label

$11.6 \%$ thiosulfate; sodium sulfite.

Log \#690019

Aluminum hardener; $\mathrm{pH}=6.9$.

Stat-King Processing Solution - Green Label

Hydroquinone, sodium sulfite in highly alkaline

solution $\left(\mathrm{OH}^{2}, \mathrm{CO}_{3}{ }^{=}\right) ; \mathrm{pH}=12.9$.

Stat-King Processing Solution - Orange Label

$35.4 \%$ thiosulfate; sodium sulfite.

Aluminum hardener; $\mathrm{pH}=5.4$.

Stat-King Processing Solution - Red Label

Contains $0.71 \%$ acetic acid, sodium sulfite, sodium acetate. $\mathrm{pH}=3.1$.

CEG

Log \#690017

CEG

Log \#690020

Stat-King Replenisher

Hydroquinone, thiosulfate, carbonate.

$\mathrm{pH}=10.6$.

Steelcote Mfg. Co., St. Louis, MO

Steelcote Concrete Floor Seal

Solvent mixture (65\%): $14 \%$ low-boiling aliphatic

$\mathrm{H}-\mathrm{C}$ mixture $\left(<120^{\circ} \mathrm{C}\right) ; 10 \%$ medium-boiling aliphatic

$\mathrm{H}-\mathrm{C}$ mixture $\left(120-160^{\circ} \mathrm{C}\right)$; $10 \%$ high-boiling aliphatic

$\mathrm{H}-\mathrm{C}$ mixture $\left(>160^{\circ} \mathrm{C}\right) ; 8 \%$ toluene, $16 \%$ p-xylene,

$10 \%$ o-xylene, $15 \%$ 1,3,5-trimethylbenzene (mesitylene),

$16 \%$ 1,2,4-trimethylbenzene (pseudocumene). No benzene, cumene, $\mathrm{p}$-cymene.

Steelcote Concrete Floor Seal

$\sim 33 \%$ linseed oil alkyd resin or soybean

Log \#720440

oil resin; $\sim 67 \%$ m-xylene + hexane/heptane as

CEG

solvents.

Steelcote EpoLux \#100 Coating E-1 White

Curing agent material is a polyamide resin with

Log \#690018

CEG

Log \#690021

CEG

Log \#680233

JPG toluene and ethylene glycol monoethyl ether as solvents. Base material is a phenolic ether resin with toluene and ethylene glycol monoethyl ether as solvents.

Log \#700755

CEG 
Steelcote EZ Strip Paint Remover

Contains water, ethyl alcohol, dichloromethane

$1 / 8 / 62$

and a $\mathrm{NaOH}$ suspension containing 0.138 equivalents/ $/$.

Steelcote EZ Strip Paint Stripper

$50 \%$ (vol) $\mathrm{CH}_{2} \mathrm{Cl}_{2}$ methylene chloride

Log \#671328

$15 \%$ (vol) propylene dichloride

35\% (vol) evap. retardant, emulsifier, and

alkaline corrosion inhibitor.

Steelcote Primer, Thioca]k 826BR

Sample - solvent

Activator - insufficient sample)

Log \#630355

630356

Primer - xylene

Thiocalk 826BR - 90\% toluene

Steelcote Stays-On Metal Primer

Isopropanol is solvent, resins are

TAL

LWB

polyurethanes.

Stress Coat

$\mathrm{CS}_{2}-56.8$ wt. $\%, 52.6$ vol. \%. Natural resin 43.2 wt. $\%, 47.4$ vol. $\%$.

Log \#641394

LWB

Log \#67699

JPG

Styrafoam

Polyethylene.

Sue Prée

Honey \& Almond Lotion

No cyanides, no peroxides; odor due to

cinnamaldehyde-type olefin hydrocarbon.

Vapor pressure @ $100^{\circ} \mathrm{F}$ - not noticeable.

Superwish - Contact Cleaner and Lubricant

Contact cleaner and lubricant; contains no

Log \#650273

carbon tetrachloride - is a mixture of freons.

Log \#673348

JPG

Sure-Rite Ditto Fluid

No ethanol; $36 \%$ by vol. MeOH; flash point below

RMT.

IWB

$10 / 7 / 59$

JE 
Tap Free

Contains 1,I,1-trichloroethane and small

$\log \# 631047$

amount of light cutting oil.

Tap Free

Solvent is $1,1,1-t$ richloroethane with small

TAL

amount of cutting oil. Solvent contains no

carbon tetrachloride.

Tap Free Drilling, Cutting, Tapping Compound

Major component is 1,1,1-trichloroethane.

Iog \#631048

TAI

Tapmaster

1,1,1-trichloroethane plus inhibitor.

Log \#630229

Log \#641526

LWB

Techni-Strip Au with Cyanide

Major constituent is $\mathrm{NaCN}$, minor constituent

Log \#714445

is $\mathrm{KCN}$, in an alkaline media.

$\mathrm{CEG}$

Teflon

(Fluon) - $\left(\mathrm{CF}_{2}=\mathrm{CF}_{2}\right)$ when heated gives off physiologically active fumes; causes illness similar to metal fume fever, sometimes pulmonary edema. Harmless at temperatures below $200^{\circ} \mathrm{C}$. Fluoride $.05 \mathrm{mg} / \mathrm{m}^{3}$.

Tellus Oil 23

No phenolic compounds present.; flash point $312^{\circ} \mathrm{F} @ 627 \mathrm{~mm} \mathrm{Hg}$.

Tempil Corporation

Pyromark Black Refractory Paint

$20+$ high boiling aliphatic hydrocarbons.

Log \#650811

LWB

Log \#772614

CEG

Tetryl

Tetralite, trinitrophenylmethylnitroamine

$1.5 \mathrm{mg} / \mathrm{m}^{3}$. Flammable explosive solid;

danagerous fire hazard. Particles can

damage eyes, upper respiratory tract and skin.

Systemic effects have been reported by tetryl

workers.

Thermofax Belt Cleaner

Flash point $51^{\circ} \mathrm{F} \mathrm{CC}$.

Thermoflex A.N.

Phenyl-beta-naphthylamine. 
Thetford Corporation, Ann Arbor, MI

Log \#730886

Aqua Ken \#03101

CEG

Contains formaldehyde, isopropanol and water.

Thiokol

Synthetic mubber prepared from 1,2-dichloroethane

$12 / 16 / 64$

and sodium polysulfide. Ref. Inv. Report 12/16/64.

A.

Thiokol Flexibilizer

A polysulfide resin.

Thixon

Syntehtic rubber.

TP

Thiokol polysulfide plasticer.

Trio

Organic paint thinner, methanol, methylene

Log \#641038

chloride, methyl-ethyl ketone plus four other

TAI

volatiles in trace amounts.

Trisec

Trichloroethylene and a cationic surface agent.

Turco - 4101

$90 \%$ trichloroethylene, $10 \%$ inhibitor (unidentified).

$7 / 10 / 63$

TAL

Turco Degreaser XL 202N

Nonhalogenated, aliphatic hydrocarbon mixture.

Log \#630060

Gas chromatogram shows at least seven components.

A petroleum distillate fraction. Boiling point $100-160^{\circ} \mathrm{C}$.

Turco Soap

$\mathrm{pH}=12$

$\log \# 630871$

$\mathrm{RM}$

Turco - Solv. Electrical Equipment Cleaner

90\% trichloroethylene, $10 \%$ inhibitor

$7 / 10 / 63$

(unidentified). Flash point $125^{\circ} \mathrm{F}$ OC.

Turpentine

Flash point $90^{\circ} \mathrm{F} \mathrm{CC}$.

$8 / 21 / 63$

MC

Tween

$20 \%$ polyoxyethylene sorbitan tristearate,

$80 \%$ polyoxyethylene sorbitan monooleate. 
Ultraset Ink 0-1867 Black

No 2-nitro propane found.

Unichrome Paint Thinner 219-ST

Diacetone alcohol in mineral spirits.

Union Carbide

Bakelite AYAC Blend 99-69

Material is a vinyl acetate resin. Products upon heating: below $500^{\circ} \mathrm{F}$ - none; at $500^{\circ} \mathrm{F}$ acetic acid; at $860^{\circ} \mathrm{F}$ - acetic acid, $\mathrm{CO}_{2}, \mathrm{CO}$, $\mathrm{H}_{2} \mathrm{O}$.

Union Carbide

ERL-4201

3,4 epoxy-6methyl cyclo hexamethyl carboxylate.

Carcinogenic, removed from market.

Union Carbide

ERI-4221

3,4 epoxy cyclo hexamethyl carboxylate.

Mildly carcinogenic on prolonged contact.

Union Rubber \& Asbestos Co., Trenton, NJ

Bestine Solvent \& Cleaner

Petroleum distillates (naphtha) 100\%. $\mathbb{N D}=1.3815$ (Dial = 18), aliphatic HC's. Boiling point $67-83^{\circ} \mathrm{C}$; extremely flammable. No aromatics, halogens, sulfides, nitrogen or oxygen. TLV 500 (67) ppm; $2000 \mathrm{mg} / \mathrm{m}^{3}$; rating moderate toxicity. Flash point less than $37^{\circ} \mathrm{F} \mathrm{OC}$; $<20^{\circ} \mathrm{F} \mathrm{CC}$; flammability $<20^{\circ} \mathrm{F}$.

Union Rubber \& Asbestos Co., Trenton, NJ Bestline Solvent Thinner

Hexane.

Union Rubber \& Asbestos Co., Trenton, NJ

Best Test Cement Dispenser

$>90 \%$ hexane; $\approx 9 \%$ rubber; < $1 \%$ benzene or toluene.

Union Rubber \& Asbestos Co., Trenton, NJ

Best Test Paper Cement

Flash point less than $41^{\circ} \mathrm{F}$.

Union Rubber \& Asbestos Co., Trenton, NJ

Best Test White Rubber Cement

$>90 \%$ hexane; $\approx 9 \%$ rubber; < $1 \%$ benzene or toluene.
Log \#772612

WE

Log \#714659

CEG

$12 / 4 / 70$

Manufacturer

Information

$12 / 4 / 70$

Manufacturer

Information

Log \#680814

LWB

Log \#700955

$\mathrm{CEG}$

Iog \#700957

CEG

Log \#700956

CEG 
U.S. Borax \& Chemical Corp.

Log \#762212

Iuron - Powdered Hand Soap

Borax, lanolin, perfume. Low toxicity

rating. $0.09 \%$ Ianolin; $\mathrm{pH}$ ( $1 \%$ solution 9.19 ).

Uresolve H.F.

95\% methyl cellosolve (2-methoxyethanol), 5\%

$\log \# 661461$ potassium carbonate $\mathrm{K}_{2} \mathrm{CO}_{3}$, trace potassium

JPG

phosphate $\mathrm{K}_{3} \mathrm{PO}_{4} ; \mathrm{pH}>11$; flash point $102^{\circ} \mathrm{F}$

$\mathrm{OC}$.

Uresolve Plus

75\% 2-methoxyethanol, 15\% DMF, 10\% DEF.

Log \#680405

JPG 
Vandex

Powdered selenium.

Van Faire Lotion

Water based lotion containing pyrilamine

Log \#661579

maleate and benzalkonium chloride (pyrilamine

LWB

maleate is an antihistiminic and benzalkonium

chloride is a surface germicide). $\mathrm{pH}=7.2$.

Van Son

Ink, Iitho Black

No 2-nitro propane found.

Log \#772613

WE

Van Wash $\mathrm{V}-120$

Contains acetone, methanol and aliphatic

hydrocarbons.

Vapco Bubble Leak Detector

$50 \%$ aqueous soap solution, $45 \%$ ethylene glycol,

LWB

and $5 \%$ ethylene diamine.

Varox

Polyethylene cross-linking agent.

Velva Sheen Floor Mop Dressing

Unsaturated petroleum distillates.

Log \#620729

Boiling point $190^{\circ}-282^{\circ} \mathrm{C}$ at $760 \mathrm{~mm}$.

LI

Miscible with Green's Allsheen.

Flash point $183^{\circ} \mathrm{F}$.

Velva-Sheen Mop Oil

Contains saturated aliphatic hydrocarbons.

Log \#772611

WE, CEG

Log \#671302

No amines, phenols or chlorinated hydrocarbons.

Vending Company Cleaning Fluid $0.0014 \% \mathrm{Ca}, 0.0175 \% \mathrm{CI}^{-}, 0.050 \% \mathrm{OCI}^{-}, 0.065 \% \mathrm{Na}^{+}$, balance is water. The solution is $0.05 \%$ Na.0CI (0.05\% available chlorine). (Chlorox is $5 \% \mathrm{NaOCl}$.)

Versene

Sodium ethylene diamine tetraacetic acid.

Vibraclean Antiseptic Hand Cleaner

Refined mineral oil and lanolin - skin conditioner -

Log \#671331

with trace triethanolamine oleate - surface active

agent, methylsalicylate - odorant, diaphene and

allantoin - antiseptics.

Log \#771195

CEG

Log \#670186

JPG

LWB

Vicap

Reversible potting compound, epoxy. 
Vicron

Marble dust.

Vikane

Ref. JOM, Aug., 1966, vol. 8, No. 8, p. 425.

Sulfuryl fluoride; use - fumigant. Gas -

slightly soluble. Toxicity rating - high;

TLV $20 \mathrm{ppm} ; \mathrm{mg} / \mathrm{m}^{3}$ (tentative suggestion).

Vi-Lan Skin Cleanser

9. $3 \% \mathrm{H}_{2} \mathrm{O}, \sim 75 \%$ paraffin hydrocarbons,

Log \#701768

$\sim 2 \%$ lanolin, $\sim 5 \%$ soap and detergents,

$<5 \%$ triethanolamine, $<1 \%$ perfume.

$\mathrm{pH}$ of $1 \%$ soln $=7.3$.

Vimasco Fire Protection Coating IA

Contains $\mathrm{Si}, \mathrm{Al}, \mathrm{Sb}, \mathrm{Mg}, \mathrm{Fe}, \mathrm{Ca}, \mathrm{Na}, \mathrm{Ti}$.

Log $\# 774090$

WE

V.O.P. -88

$\mathrm{N}, \mathrm{N}$-dioctyl-p-phenylenediamine. 
Wasco Type Ditto Fluid

See original for data.

$10 / 7 / 59$

JE

Washorite \#2

Contains aliphatic hydrocarbon and

Log \#772609

tetra chloroethylene.

Wayclean Copper Brightener

Contains anionic synthetic surfactant, sodium chloride, sodium bisulfate. Acidity is due to high percentage of sodium bisulfate. Contains a sodium acid phosphate as a corrosion inhibitor, $\mathrm{pH}$ of $1 \%$ soln $=2.9$.

Webtex Adhesive

Flash point $71^{\circ} \mathrm{F}$ CC.

Weedon WD-4 Bug Killer

Contains as functional groups: ethyl ester, aliphatic acid, a polychloro phenol, phosphate ester, petroleum oil.

Welding Rod \#7018

Flux contains high amounts of fluorides, chlorides and borates.

WE, CEG

Log \#691996

$\mathrm{CEG}$

Iog \#691901 CEG

Log \#761110 CEG

Wellborn Paint Co.

Iog \#680361

Wellborn Thinner

$14.5 \%$ isopropanol, $26.7 \%$ acetone, $3.7 \%$

JPG n-hexane, $9.7 \%$ MTBK, $45.3 \%$ toluene. Flash point $31^{\circ} \mathrm{F}$ OC.

Wesgro $A L-500$

$\mathrm{CO}_{2}$ ethylene and trace of formaldehyde was given off at $350^{\circ} \mathrm{C}$.

Log \#751253 CEG

Wesgro AL-995

$\mathrm{CO}_{2}$ ethylene and trace of formaldehyde

Log \#751252

CEG

was given off at $350^{\circ} \mathrm{C}$.

West Chemical Co.

$\log \# 672434$

West Lan-O-Kleen

JPG

$3 \%$ lanolin, minor borax, minor silicates, spectral purity - fair, $\mathrm{pH}$ of $1 \%$ solution (slurry) $=9.6 ; \%$ insoluble in $1 \%$ slurry $-55 \%$. Odor - soapy, mild; particle size uniformity poor; dust generation when poured - none. 
West Chemical Co.

Log \#680357

Lan-O-Kleen Plus

3.3\% lanolin, $54 \% \mathrm{H}_{2} \mathrm{O}$ - insoluble, $\mathrm{pH}$ of $5 \%$

soln $=9.2$, contains fluorescein coloring

agent. Label specifies bithionol content

$2,2^{\prime}$-thiobis $\left(4,6\right.$-dichlorophenyl) $\mathrm{HoCl}_{2} \phi \mathrm{s} \phi \mathrm{Cl}_{2} \mathrm{OH}$.

West Plus Hand Cleaner

$23.5 \% \mathrm{H}_{2} \mathrm{O}, \sim 70 \%$ paraffin hydrocarbons, $\sim 5 \%$

Log \#701764 soap and detergent, $<5 \%$ triethanolamine, $<1 \%$ perfume, no lanolin detected. $\mathrm{pH}$ of $1 \%$ $\operatorname{soln}=5.8$.

West Superlode

Material is an aliphatic hydrocarbon oil.

Log \#772716

CEG

Weston Industries, Inc., Newark, NJ

Weston Statnul

Contains water and isopropanol, $\mathrm{pH}=3.6$.

Log \#751536

CEG

Wirth Co. Inc., Oakland, CA

Correctette Opaque White Correction Fluid

Log \#680815

Solvent - toluene + white pigment;

LWB

thinner - toluene. TLV $200(67) \mathrm{ppm} ; \mathrm{mg} / \mathrm{m}^{3}$.

Wirth Co. Inc., Haward, CA

Correctette Thinner CT2

Contains 1,1,1-trichloroethane.

Log \#780413

CEG

White A.R. Brazive \#3

Log \#715102

$0.99 \% \mathrm{SiO}_{2}$.

CEG

White Cloud Correction Fluid

Solvent contains $100 \%$ toluene.

Log \#630145

$\mathrm{RM}$

Wingstay 100

Alkyl-aryl-amine.

Wonder Flux Entectic

$\mathrm{Na}$, Li, Ca, Al, B, K, Si; $1.1 \%$ fluoride

Log \#660564

(eutectic).

$\mathrm{EG}$

Wyandotte Flote

Paint water wall coagulating agent.

Wyandotte Quadrol

Epoxy curing retardant; $\mathbb{N}^{\prime}, \mathbb{N}^{\prime}, \mathbb{N}^{\prime}, \mathbb{N}^{\prime}$-tetrakis

(2-hydroxypropyl)-ethylenediamine. 
Wyandotte Stainless Steel Polish

Log \#732416

Liquid phase - mineral oil; propellant - freon;

LWB

slight trace of odorant. 
Xerox

914 Developer

Carbon black on sea sand.

Xerox

Film Remover

Isopropyl alcohol. Flash point $11.7^{\circ} \mathrm{C}\left(53^{\circ} \mathrm{F}\right)$.

Xerox

Film Remover

100\% iso-propanol (container label says "contains isopropanol").

Xerox

Film Remover

Is opropanol.

Xerox

"Flo-Set" Fluid

$100 \%$ trichloroethylene.

Xerox

600 Toner

100\% carbon black.

Xerox

914 Toner

Black thermoplastic resin.

Xerox

1824 Toner

Carbon black with trace of aluminum.

Xerox

2400 Toner

Acrylic resin with carbon black filler.

XI Plate Etch

Aqueous solution $\mathrm{pH}=4.5$; no organics.
Log \#641519

LWB

Iog \#620092

Jack

Log \#630501

TAI

$\log \# 641517$

Log \#630387

TAI

$1 / 18 / 72$

CEG

Log \#641520

LWB

$1 / 18 / 72$

$\mathrm{CEG}$

Log \#690259

$\mathrm{CEG}$

Log \#772690

CEG 
Yardney Silvercel Electrolyte $R$

Log \#680246

Solution is $10 \mathrm{~N} \mathrm{KOH}$; $\mathrm{pH}=14$.

LWB

$-80-$ 
Zapon Thinner Grade 101-8A

$55 \%$ ethyl acetate, $35 \%$ toluene, $5 \%$ butyl

$\log \# 681897$ acetate, $5 \%$ xylene.

Zepco

Zep F-7 Powdered Hand Cleaner Code \#5

$0.045 \%$ lanolin, major borax, major silicates, poor spectral purity, $\mathrm{pH}$ of $1 \%$ slurry - $60 \%$.

Odor - minty, strong; particle size uniformity poor; dust generation when poured - much.

Zep Mfg. Co.

Zep Cleansing Solution

Emission spectra shows the elements $\mathrm{Na}, \mathrm{Al}$, Si, $\mathrm{pH}=14$. Probably sodium silicates, sodium aluminum silicates.

Zip Carb Cleaner Mixture of cresols, alcohol, organic acids, Log \#714814 chlorinated hydrocarbons and water. Not miscible in water.

Zorbal Sweeping Compound $18.62 \%$ free silica.

Log \#761598 CEG

Log \#672715 JPG

$\mathrm{CEG}$

Zorball

Calcined mineral (diatomaceous earth).

Log \#713438 LWB

Log \#660517 LWB

Zyglo Developer Type ZP-9

Contains $\approx 70 \%$ trichloroethylene and $\approx 30 \%$

Log \#730771 acetone or methyl ketone as solvents. Also CEG contains a fluorescent dye. 
DISTRIBUTION:

UC-4I Health and Safety (TID-4500-R66) (25I)

DOE Library

Mail Station G-043

Washington, DC 20545

DOE Albuquerque Operations Office

P. O. Box 5400

Albuquerque, IM 87115

DOE Sandia Area Office

P. O. Box 5800

Albuquerque, NM 87185

Los Alamos Scientific Laboratory

P.O. Box 1663

Los Alamos, NM 87544

Attn: Evan Campbell, H-5

G. D. Ware

Industrial Hygiene Consultant

Western Electric

222 Broadway

New York, NY 10038

3000 R. B. Powell

3300 P. B. Mossman (4)

3310 W. D. Burnett

3311 L. W. Brewer

3311 W. Einfeld

3311 C. E. Gray (10)

3311 J. W. McKelvey

3311 D. R. Parker

3311 C. J. Pigg

3311 C. P. Skillern

3311 W. E. Stocum

3312 G. E. Tucker, Jr.

3313 A. I. Stanley

3322 J. W. Ewing

8252 P. K. LoveII

3141 T. I. Werner (5)

3151 W. L. Garner (3) For DOE/TIC (Unlimited Release)

8266 E. A. Aas 